\title{
Multiobjective Fractional Programming Involving Generalized Semilocally V-Type I-Preinvex and Related Functions
}

\author{
Hachem Slimani ${ }^{1}$ and Shashi Kant Mishra ${ }^{2}$ \\ ${ }^{1}$ LaMOS Research Unit, Computer Science Department, University of Bejaia, 06000 Bejaia, Algeria \\ ${ }^{2}$ Department of Mathematics, Faculty of Science, Banaras Hindu University, Varanasi 221005, India \\ Correspondence should be addressed to Hachem Slimani; haslimani@gmail.com
}

Received 12 January 2014; Revised 5 April 2014; Accepted 5 April 2014; Published 5 May 2014

Academic Editor: Ram U. Verma

Copyright (c) 2014 H. Slimani and S. K. Mishra. This is an open access article distributed under the Creative Commons Attribution License, which permits unrestricted use, distribution, and reproduction in any medium, provided the original work is properly cited.

\begin{abstract}
We study a nonlinear multiple objective fractional programming with inequality constraints where each component of functions occurring in the problem is considered semidifferentiable along its own direction instead of the same direction. New Fritz John type necessary and Karush-Kuhn-Tucker type necessary and sufficient efficiency conditions are obtained for a feasible point to be weakly efficient or efficient. Furthermore, a general Mond-Weir dual is formulated and weak and strong duality results are proved using concepts of generalized semilocally V-type I-preinvex functions. This contribution extends earlier results of Preda (2003), Mishra et al. (2005), Niculescu (2007), and Mishra and Rautela (2009), and generalizes results obtained in the literature on this topic.
\end{abstract}

\section{Introduction}

Because of many practical optimization problems where the objective functions are quotients of two functions, multiobjective fractional programming has received much interest and has grown significantly in different directions in the setting of efficiency conditions and duality theory these later years. The field of multiobjective fractional optimization has been naturally enriched by the introductions and applications of various types of convexity theory, with and without differentiability assumptions, and in the framework of symmetric duality, variational problems, minimax programming, continuous time programming, and so forth. More specifically, works in the area of nonsmooth setting can be found in Chen [1], Kim et al. [2], Kuk et al. [3], Mishra and Rautela [4], Mishra et al. [5], Niculescu [6], Preda [7], and Soleimani-damaneh [8]. Efficiency conditions and duality models for multiobjective fractional subset programming problems are studied by Preda et al. [9], Verma [10], and Zalmai [11-13]. Higher order duality in multiobjective fractional programming is discussed in Gulati and Geeta [14] and Suneja et al. [15]. Solving nonlinear multiobjective fractional programming problems by a modified objective function method is the subject matter of Antczak [16]. Further works on multiobjective fractional programming are established by Chinchuluun et al. [17], J.-C. Liu and C.-Y. Liu [18], Mishra et al. [19], Verma [20], Zhang and Wu [21], and others.

The common point in all of these developments is the convexity theory that does not stop extending itself in different directions with new variants of generalized convexity and various applications to nonlinear programming problems in different settings. The concept of invexity introduced by Hanson [22] is a generalization of convexity which has received much interest these later years, and many advances in the theory and practice have been established using this concept and its extensions. In practice, recently Dinuzzo et al. [23] have obtained some kernel function in machine learning which is not quasiconvex (and hence also neither convex nor pseudoconvex), but it is invex. Nickisch and Seeger [24] have studied a multiple kernel learning problem and have used the invexity to deal with the optimization which is nonconvex. The concept of semilocally convex functions was introduced by Ewing [25] and was further extended to semilocally quasiconvex, semilocally pseudoconvex functions by Kaul and Kaur [26, 27]. Other generalizations of semilocally convex functions and their properties were investigated in 
Mishra and Rautela [4], Mishra et al. [5], Niculescu [6], Preda [7, 28], Preda and Stancu-Minasian [29], Preda et al. [30], and Stancu-Minasian [31].

In Preda [7], necessary and sufficient efficiency conditions for a nonlinear fractional multiple objective programming problem are obtained involving $\eta$-semidifferentiable functions. Furthermore, a general dual was formulated and duality results were proved using concepts of generalized semilocally preinvex functions. Thus, results of Preda [28], Preda and Stancu-Minasian [29], and Preda et al. [30] were generalized. Mishra et al. [5] extended the issues of Preda [7] to the case of semilocally type I and related functions, generalizing results of Preda [7] and Stancu-Minasian [31]. Niculescu [6] extended the work of Mishra et al. [5] by using concepts of generalized $\rho$-semilocally type I-preinvex functions. Mishra and Rautela [4] extended the works of Mishra et al. [5] and Preda [7] to the case of semilocally type I univex and related functions.

By considering the invexity with respect to different $\left(\eta_{i}\right)_{i}$ (each function occurring in the studied problem is considered with respect to its own function $\eta_{i}$ instead of the same function $\eta$ ), Slimani and Radjef [32-34] have obtained necessary and sufficient optimality/efficiency conditions and duality results for nonlinear scalar and (nondifferentiable) multiobjective problems. Ahmad [35] has considered a nondifferentiable multiobjective problem and, by using generalized univexity with respect to different $\left(\eta_{i}\right)_{i}$, he has obtained efficiency conditions and duality results. Arana-Jiménez et al. [36] have used the concept of semidirectionally differentiable functions introduced in [34] to derive characterizations of solutions and duality results by means of generalized pseudoinvexity for nondifferentiable multiobjective programming.

In this paper, motivated by the work of Slimani and Radjef [34], we define semilocally vector-type I problems, where each component of the objective and constraint functions is semidifferentiable along its own direction instead of the same direction. Then we consider necessary and sufficient efficiency conditions for a nonlinear fractional multiple objective programming problem involving semidifferentiable functions. Furthermore, we formulate a general Mond-Weir dual and prove duality results using concepts of generalized semilocally V-type I-preinvex and related functions. Thus, we extend the works of Mishra and Rautela [4], Mishra et al. [5], Niculescu [6], and Preda [7] and generalize results obtained in the literature on this topic.

\section{Preliminaries and Definitions}

The following conventions for equalities and inequalities will be used. If $x=\left(x_{1}, \ldots, x_{n}\right)$ and $y=\left(y_{1}, \ldots, y_{n}\right) \in \mathbb{R}^{n}$, then $x=y \Leftrightarrow x_{i}=y_{i}, i=1, \ldots, n ; x<y \Leftrightarrow x_{i}<y_{i}, i=1, \ldots, n$; $x \leqq y \Leftrightarrow x_{i} \leqq y_{i}, i=1, \ldots, n ; x \leq y \Leftrightarrow x \leqq y$ and $x \neq y$.

We also denote by $\mathbb{R}_{\geqq}^{q}$ (resp., $\mathbb{R}_{\geq}^{q}$ or $\mathbb{R}_{>}^{q}$ ) the set of vectors $y \in \mathbb{R}^{q}$ with $y \geqq 0$ (resp., $y \geq 0$ or $y>0$ ).

Let $D \subseteq \mathbb{R}^{n}$ be a set and $\eta: D \times D \rightarrow \mathbb{R}^{n}$ a vector application. We say that $D$ is $\eta$-invex at $x_{0} \in D$ if $x_{0}+$ $\lambda \eta\left(x, x_{0}\right) \in D$ for any $x \in D$ and $\lambda \in[0,1]$. We say that the set $D$ is $\eta$-invex if $D$ is $\eta$-invex at any $x_{0} \in D$.

Definition 1 (see [7]). We say that the set $D \subseteq \mathbb{R}^{n}$ is an $\eta$ locally star-shaped set at $x_{0}, x_{0} \in D$, if, for any $x \in D$, there exists $0<a_{\eta}\left(x, x_{0}\right) \leqq 1$ such that $x_{0}+\lambda \eta\left(x, x_{0}\right) \in D$ for any $\lambda \in\left[0, a_{\eta}\left(x, x_{0}\right)\right]$.

Definition 2 (see [7]). Let $f: D \rightarrow \mathbb{R}^{N}$ be a function, where $D \subseteq \mathbb{R}^{n}$ is an $\eta$-locally star-shaped set at $x_{0} \in D$. We say that $f$ is $\eta$-semidifferentiable at $x_{0}$ if $(d f)^{+}\left(x_{0}, \eta\left(x, x_{0}\right)\right)$ exists for each $x \in D$, where

$$
\begin{aligned}
(d f)^{+} & \left(x_{0}, \eta\left(x, x_{0}\right)\right) \\
& =\lim _{\lambda \rightarrow 0^{+}} \frac{1}{\lambda}\left[f\left(x_{0}+\lambda \eta\left(x, x_{0}\right)\right)-f\left(x_{0}\right)\right]
\end{aligned}
$$

(the right derivative of $f$ at $x_{0}$ along the direction $\eta\left(x, x_{0}\right)$ ). If $f$ is $\eta$-semidifferentiable at any $x_{0} \in D$, then $f$ is said to be $\eta$-semidifferentiable on $D$.

Note that a function which is $\eta$-semidifferentiable at $x_{0}$ is not necessarily directionally differentiable at this point (see [34]). Slimani and Radjef [34] have considered the functions whose directional derivatives exist and are finite in some directions (not necessarily in all directions) and they called them semidirectionally differentiable functions. This class of functions, where no assumptions on continuity are needed, is an extension of locally Lipschitz functions.

Definition 3 (see [37]). A function $f: D \rightarrow \mathbb{R}^{N}$ is a convexlike function if, for any $x, y \in D$ and $0 \leqq \lambda \leqq 1$, there exists $z \in D$ such that

$$
f(z) \leqq \lambda f(x)+(1-\lambda) f(y) .
$$

We consider the following multiobjective fractional optimization problem:

$$
\begin{array}{cl}
\text { (VFP) Minimize } & \frac{f(x)}{g(x)}=\left(\frac{f_{1}(x)}{g_{1}(x)}, \ldots, \frac{f_{N}(x)}{g_{N}(x)}\right), \\
\text { subject to } & h_{j}(x) \leqq 0, \quad j=1,2, \ldots, k,
\end{array}
$$

where $f_{i}, g_{i}, h_{j}: D \rightarrow \mathbb{R}, i \in \mathcal{N}=\{1,2, \ldots, N\}, j \in$ $K=\{1,2, \ldots, k\}$ with $D$ is a nonempty subset of $\mathbb{R}^{n}$, and $f_{i}(x) \geqq 0, g_{i}(x)>0$ for all $x \in D$ and each $i \in \mathcal{N}$. Let $f=\left(f_{1}, \ldots, f_{N}\right), g=\left(g_{1}, \ldots, g_{N}\right)$, and $h=\left(h_{1}, \ldots, h_{k}\right)$.

We put $X=\{x \in D: h(x) \leqq 0\}$ as the set of all feasible solutions of VFP. For $x_{0} \in D$, we denote by $J\left(x_{0}\right)$ the set $\left\{j \in K: h_{j}\left(x_{0}\right)=0\right\}$, where $J=\left|J\left(x_{0}\right)\right|$ is the cardinal of set $J\left(x_{0}\right)$, and by $\widetilde{J}\left(x_{0}\right)$ (resp., $\left.\bar{J}\left(x_{0}\right)\right)$ the set $\left\{j \in K: h_{j}\left(x_{0}\right)<\right.$ 0 (resp., $\left.\left.h_{j}\left(x_{0}\right)>0\right)\right\}$. We have $J\left(x_{0}\right) \cup \widetilde{J}\left(x_{0}\right) \cup \bar{J}\left(x_{0}\right)=K$ and if $x_{0} \in X, \bar{J}\left(x_{0}\right)=\emptyset$.

For such optimization problems, minimization means obtaining (weakly) efficient solutions in the following sense. 
Definition 4. A point $x_{0} \in X$ is said to be a weakly efficient solution of the problem VFP, if there exists no $x \in X$ such that

$$
\frac{f_{i}(x)}{g_{i}(x)}<\frac{f_{i}\left(x_{0}\right)}{g_{i}\left(x_{0}\right)}, \quad \forall i \in \mathcal{N} .
$$

Definition 5. A point $x_{0} \in X$ is said to be an efficient solution of the problem VFP, if there exists no $x \in X$ such that for some $p \in \mathcal{N}$

$$
\frac{f_{p}(x)}{g_{p}(x)}<\frac{f_{p}\left(x_{0}\right)}{g_{p}\left(x_{0}\right)}, \quad \frac{f_{i}(x)}{g_{i}(x)} \leqq \frac{f_{i}\left(x_{0}\right)}{g_{i}\left(x_{0}\right)}, \quad \forall i \in \mathcal{N}, i \neq p .
$$

Slimani and Radjef [34] considered semidirectionally differentiable functions and extended the $d$-invexity of Ye [38] by introducing new concepts of generalized $d_{I}$-invexity in which each component of the objective and constraint functions is directionally differentiable in its own direction $d_{i}$ instead of the same direction $d$. In the same way, we define semilocally vector-type I problems, where each component of the objective and constraint functions is semidifferentiable along its own direction $\eta_{i}, \theta_{i}$, or $\delta_{j}$ instead of the same direction $\eta$.

Definition 6. Let $\eta_{i}: X \times D \rightarrow \mathbb{R}^{n}, \theta_{i}: X \times D \rightarrow \mathbb{R}^{n}, i \in \mathcal{N}$, and $\phi_{j}: X \times D \rightarrow \mathbb{R}^{n}, j \in K$, be vector functions. We say that the problem VFP is semilocally $V$-type I-preinvex at $x_{0} \in D$ with respect to $\left(\eta_{i}\right)_{i \in \mathcal{N}},\left(\theta_{i}\right)_{i \in \mathscr{N}}$, and $\left(\phi_{j}\right)_{j \in K}$, if, for all $x \in X$, we have

$$
\begin{array}{cc}
f_{i}(x)-f_{i}\left(x_{0}\right) \geqq\left(d f_{i}\right)^{+}\left(x_{0}, \eta_{i}\left(x, x_{0}\right)\right), & \forall i \in \mathcal{N}, \\
g_{i}(x)-g_{i}\left(x_{0}\right) \leqq\left(d g_{i}\right)^{+}\left(x_{0}, \theta_{i}\left(x, x_{0}\right)\right), & \forall i \in \mathcal{N}, \\
-h_{j}\left(x_{0}\right) \geqq\left(d h_{j}\right)^{+}\left(x_{0}, \phi_{j}\left(x, x_{0}\right)\right), & \forall j \in K .
\end{array}
$$

If the inequalities in (6) are strict (whenever $x \neq x_{0}$ ), we say that VFP is semistrictly semilocally $V$-type I-preinvex at $x_{0}$ with respect to $\left(\eta_{i}\right)_{i \in \mathcal{N}},\left(\theta_{i}\right)_{i \in \mathscr{N}}$, and $\left(\phi_{j}\right)_{j \in K}$.

Definition 7. Let $\eta_{i}: X \times D \rightarrow \mathbb{R}^{n}, \theta_{i}: X \times D \rightarrow \mathbb{R}^{n}, i \in \mathcal{N}$, and $\phi_{j}: X \times D \rightarrow \mathbb{R}^{n}, j \in K$, be vector functions. We say that the problem VFP is semilocally pseudo quasi-V-type I-preinvex at $x_{0} \in D$ with respect to $\left(\eta_{i}\right)_{i \in \mathcal{N}},\left(\theta_{i}\right)_{i \in \mathscr{N}}$, and $\left(\phi_{j}\right)_{j \in K}$, if, for some vectors $\mu \in \mathbb{R}_{\geqq}^{N}, \lambda \in \mathbb{R}_{\geqq}^{N}$, and $\delta \in \mathbb{R}_{\geqq}^{k}$, we have

$$
\begin{gathered}
\sum_{i=1}^{N} \mu_{i}\left[\left(d f_{i}\right)^{+}\left(x_{0}, \eta_{i}\left(x, x_{0}\right)\right)\right. \\
\left.\quad-\lambda_{i}\left(d g_{i}\right)^{+}\left(x_{0}, \theta_{i}\left(x, x_{0}\right)\right)\right] \geqq 0 \\
\Longrightarrow \sum_{i=1}^{N} \mu_{i}\left[f_{i}(x)-\lambda_{i} g_{i}(x)\right] \\
\geqq \sum_{i=1}^{N} \mu_{i}\left[f_{i}\left(x_{0}\right)-\lambda_{i} g_{i}\left(x_{0}\right)\right], \quad \forall x \in X,
\end{gathered}
$$

$$
\sum_{j=1}^{k} \delta_{j} h_{j}\left(x_{0}\right) \geqq 0 \Longrightarrow \sum_{j=1}^{k} \delta_{j}\left(d h_{j}\right)^{+}\left(x_{0}, \phi_{j}\left(x, x_{0}\right)\right) \leqq 0,
$$

$\forall x \in X$.

If the second (implied) inequality in (9) is strict $\left(x \neq x_{0}\right)$, we say that VFP is semilocally strictly pseudo quasi-V-type Ipreinvex at $x_{0}$ with respect to $\left(\eta_{i}\right)_{i \in \mathscr{N}},\left(\theta_{i}\right)_{i \in \mathcal{N}}$, and $\left(\phi_{j}\right)_{j \in K}$.

Definition 8. Let $\eta_{i}: X \times D \rightarrow \mathbb{R}^{n}, \theta_{i}: X \times D \rightarrow \mathbb{R}^{n}, i \in \mathcal{N}$, and $\phi_{j}: X \times D \rightarrow \mathbb{R}^{n}, j \in K$, be vector functions. We say that the problem VFP is semilocally quasi pseudo-V-type I-preinvex at $x_{0} \in D$ with respect to $\left(\eta_{i}\right)_{i \in \mathcal{N}},\left(\theta_{i}\right)_{i \in \mathcal{N}}$, and $\left(\phi_{j}\right)_{j \in K}$, if, for some vectors $\mu \in \mathbb{R}_{\geqq}^{N}, \lambda \in \mathbb{R}_{\geqq}^{N}$, and $\delta \in \mathbb{R}_{\geqq}^{k}$, we have

$$
\begin{aligned}
& \sum_{i=1}^{N} \mu_{i}\left[f_{i}(x)-\lambda_{i} g_{i}(x)\right] \\
& \leqq \sum_{i=1}^{N} \mu_{i}\left[f_{i}\left(x_{0}\right)-\lambda_{i} g_{i}\left(x_{0}\right)\right] \\
& \Longrightarrow \sum_{i=1}^{N} \mu_{i}\left[\left(d f_{i}\right)^{+}\left(x_{0}, \eta_{i}\left(x, x_{0}\right)\right)\right. \\
& \left.\quad-\lambda_{i}\left(d g_{i}\right)^{+}\left(x_{0}, \theta_{i}\left(x, x_{0}\right)\right)\right] \leqq 0, \quad \forall x \in X, \\
& \sum_{j=1}^{k} \delta_{j}\left(d h_{j}\right)^{+}\left(x_{0}, \phi_{j}\left(x, x_{0}\right)\right) \geqq 0 \Longrightarrow \sum_{j=1}^{k} \delta_{j} h_{j}\left(x_{0}\right) \leqq 0
\end{aligned}
$$

If the second (implied) inequality in (12) is strict $\left(x \neq x_{0}\right)$, we say that VFP is semilocally quasi strictly pseudo-V-type Ipreinvex at $x_{0}$ with respect to $\left(\eta_{i}\right)_{i \in \mathscr{N}},\left(\theta_{i}\right)_{i \in \mathcal{N}}$, and $\left(\phi_{j}\right)_{j \in K}$.

Definition 9. Let $\eta_{i}: X \times D \rightarrow \mathbb{R}^{n}, \theta_{i}: X \times D \rightarrow \mathbb{R}^{n}, i \in \mathcal{N}$, and $\phi_{j}: X \times D \rightarrow \mathbb{R}^{n}, j \in K$, be vector functions. We say that the problem VFP is semilocally extendedly pseudo partially quasi-V-type I-preinvex at $x_{0} \in D$ with respect to $\left\{\left(\eta_{i}\right)_{i \in \mathcal{N}},\left(\theta_{i}\right)_{i \in \mathcal{N}},\left(\phi_{j}\right)_{j \in J_{0}}\right\}$ and $\left\{\left(\phi_{j}\right)_{j \in J_{s}}, s=1,2, \ldots, \alpha\right\}$ such that $\alpha \geqq 1, J_{s} \cap J_{t}=\emptyset$ for $s \neq t$ and $\bigcup_{s=0}^{\alpha} J_{s}=K$, if, for some vectors $\mu \in \mathbb{R}_{\geqq}^{N}, \lambda \in \mathbb{R}_{\geqq}^{N}$, and $\delta \in \mathbb{R}_{\geqq}^{k}$, we have

$$
\begin{gathered}
\sum_{i=1}^{N} \mu_{i}\left[\left(d f_{i}\right)^{+}\left(x_{0}, \eta_{i}\left(x, x_{0}\right)\right)-\lambda_{i}\left(d g_{i}\right)^{+}\left(x_{0}, \theta_{i}\left(x, x_{0}\right)\right)\right] \\
\quad+\sum_{j \in J_{0}} \delta_{j}\left(d h_{j}\right)^{+}\left(x_{0}, \phi_{j}\left(x, x_{0}\right)\right) \geqq 0 \\
\Longrightarrow \sum_{i=1}^{N} \mu_{i}\left[f_{i}(x)-\lambda_{i} g_{i}(x)\right]+\sum_{j \in J_{0}} \delta_{j} h_{j}(x) \\
\geqq \sum_{i=1}^{N} \mu_{i}\left[f_{i}\left(x_{0}\right)-\lambda_{i} g_{i}\left(x_{0}\right)\right]+\sum_{j \in J_{0}} \delta_{j} h_{j}\left(x_{0}\right),
\end{gathered}
$$




$$
\begin{array}{r}
\sum_{j \in J_{s}} \delta_{j} h_{j}\left(x_{0}\right) \geqq 0 \Longrightarrow \sum_{j \in J_{s}} \delta_{j}\left(d h_{j}\right)^{+}\left(x_{0}, \phi_{j}\left(x, x_{0}\right)\right) \leqq 0, \\
\forall x \in X, \quad s=1,2, \ldots, \alpha .
\end{array}
$$

If the second (implied) inequality in (13) is strict $\left(x \neq x_{0}\right)$, we say that VFP is semilocally strictly extendedly pseudo partially quasi-V-type I-preinvex at $x_{0}$ with respect to $\left\{\left(\eta_{i}\right)_{i \in \mathcal{N}}\right.$, $\left.\left(\theta_{i}\right)_{i \in \mathcal{N}},\left(\phi_{j}\right)_{j \in J_{0}}\right\}$ and $\left\{\left(\phi_{j}\right)_{j \in J_{s}}, s=1,2, \ldots, \alpha\right\}$. If, for all $i \in$ $\mathcal{N}, \theta_{i}=\eta_{i}$, we say that VFP is semilocally (strictly) extendedly pseudo partially quasi-V-type I-preinvex at $x_{0}$ with respect to $\left\{\left(\eta_{i}\right)_{i \in \mathscr{N}},\left(\phi_{j}\right)_{j \in J_{0}}\right\}$ and $\left\{\left(\phi_{j}\right)_{j \in J_{s}}, s=1,2, \ldots, \alpha\right\}$.

Remark 10. In Definition 9 if $J_{0}=\emptyset$ and $\alpha=1$, then the concept of semilocally (strictly) extendedly pseudo partially quasi-V-type I-preinvexity reduces to the concept of semilocally (strictly) pseudo quasi-V-type I-preinvexity given in Definition 7.

Definition 11. Let $\eta_{i}: X \times D \rightarrow \mathbb{R}^{n}, \theta_{i}: X \times D \rightarrow \mathbb{R}^{n}, i \in$ $\mathcal{N}$, and $\phi_{j}: X \times D \rightarrow \mathbb{R}^{n}, j \in K$, be vector functions. We say that the problem VFP is semilocally extendedly quasi partially pseudo-V-type I-preinvex at $x_{0} \in D$ with respect to $\left\{\left(\eta_{i}\right)_{i \in \mathscr{N}}\right.$, $\left.\left(\theta_{i}\right)_{i \in \mathcal{N}},\left(\phi_{j}\right)_{j \in J_{0}}\right\}$ and $\left\{\left(\phi_{j}\right)_{j \in J_{s}}, s=1,2, \ldots, \alpha\right\}$ such that $\alpha \geqq$ $1, J_{s} \cap J_{t}=\emptyset$ for $s \neq t$ and $\bigcup_{s=0}^{\alpha} J_{s}=K$, if, for some vectors $\mu \in \mathbb{R}_{\geqq}^{N}, \lambda \in \mathbb{R}_{\geqq}^{N}$, and $\delta \in \mathbb{R}_{\geqq}^{k}$, we have

$$
\begin{aligned}
& \sum_{i=1}^{N} \mu_{i}\left[f_{i}(x)-\lambda_{i} g_{i}(x)\right]+\sum_{j \in J_{0}} \delta_{j} h_{j}(x) \\
& \leqq \sum_{i=1}^{N} \mu_{i}\left[f_{i}\left(x_{0}\right)-\lambda_{i} g_{i}\left(x_{0}\right)\right]+\sum_{j \in J_{0}} \delta_{j} h_{j}\left(x_{0}\right) \\
& \Longrightarrow \sum_{i=1}^{N} \mu_{i}\left[\left(d f_{i}\right)^{+}\left(x_{0}, \eta_{i}\left(x, x_{0}\right)\right)-\lambda_{i}\left(d g_{i}\right)^{+}\left(x_{0}, \theta_{i}\left(x, x_{0}\right)\right)\right] \\
& \quad+\sum_{j \in J_{0}} \delta_{j}\left(d h_{j}\right)^{+}\left(x_{0}, \phi_{j}\left(x, x_{0}\right)\right) \leqq 0, \quad \forall x \in X,
\end{aligned}
$$$$
\sum_{j \in J_{s}} \delta_{j}\left(d h_{j}\right)^{+}\left(x_{0}, \phi_{j}\left(x, x_{0}\right)\right) \geqq 0 \Longrightarrow \sum_{j \in J_{s}} \delta_{j} h_{j}\left(x_{0}\right) \leqq 0,
$$

$$
\forall x \in X, \quad s=1,2, \ldots, \alpha .
$$

If the second (implied) inequality in (16) is strict $\left(x \neq x_{0}\right)$, we say that VFP is semilocally extendedly quasi strictly partially pseudo-V-type I-preinvex at $x_{0}$ with respect to $\left\{\left(\eta_{i}\right)_{i \in \mathcal{N}}\right.$, $\left.\left(\theta_{i}\right)_{i \in \mathscr{N}},\left(\phi_{j}\right)_{j \in J_{0}}\right\}$ and $\left\{\left(\phi_{j}\right)_{j \in J_{s}}, s=1,2, \ldots, \alpha\right\}$. If, for all $i \in$ $\mathcal{N}, \theta_{i}=\eta_{i}$, we say that VFP is semilocally extendedly quasi (strictly) partially pseudo-V-type I-preinvex at $x_{0}$ with respect to $\left\{\left(\eta_{i}\right)_{i \in \mathscr{N}},\left(\phi_{j}\right)_{j \in J_{0}}\right\}$ and $\left\{\left(\phi_{j}\right)_{j \in J_{s}}, s=1,2, \ldots, \alpha\right\}$.

Remark 12. In Definition 11 if $J_{0}=\emptyset$ and $\alpha=1$, then the concept of semilocally extendedly quasi (strictly) partially pseudo-V-type I-preinvexity reduces to the concept of semilocally quasi (strictly) pseudo-V-type I-preinvexity given in Definition 8.

\section{Necessary Efficiency Conditions}

To prove necessary conditions for VFP, we need to prove the following lemma.

Lemma 13. Suppose that

(i) $x_{0}$ is a (local) weakly efficient solution for VFP;

(ii) $h_{j}$ is continuous at $x_{0}$ for $j \in \widetilde{J}\left(x_{0}\right)$ and there exist vector functions $\eta_{i}: X \times D \rightarrow \mathbb{R}^{n}, \theta_{i}: X \times D \rightarrow$ $\mathbb{R}^{n}, i \in \mathcal{N}$, and $\phi_{j}: X \times D \rightarrow \mathbb{R}^{n}, j \in J\left(x_{0}\right)$, which satisfy at $x_{0}$ with respect to $\eta: X \times D \rightarrow \mathbb{R}^{n}$ the following inequalities:

$$
\begin{array}{r}
\left(d f_{i}\right)^{+}\left(x_{0}, \eta\left(x, x_{0}\right)\right) \leqq\left(d f_{i}\right)^{+}\left(x_{0}, \eta_{i}\left(x, x_{0}\right)\right), \\
\forall x \in X, \quad \forall i \in \mathcal{N}, \\
\left(d g_{i}\right)^{+}\left(x_{0}, \eta\left(x, x_{0}\right)\right) \geqq\left(d g_{i}\right)^{+}\left(x_{0}, \theta_{i}\left(x, x_{0}\right)\right), \\
\forall x \in X, \quad \forall i \in \mathcal{N}, \\
\left(d h_{j}\right)^{+}\left(x_{0}, \eta\left(x, x_{0}\right)\right) \leqq\left(d h_{j}\right)^{+}\left(x_{0}, \phi_{j}\left(x, x_{0}\right)\right), \\
\forall x \in X, \quad \forall j \in J\left(x_{0}\right) .
\end{array}
$$

Then the system of inequalities

$$
\begin{gathered}
\left(d f_{i}\right)^{+}\left(x_{0}, \eta_{i}\left(x, x_{0}\right)\right)<0, \quad i \in \mathcal{N}, \\
\left(d g_{i}\right)^{+}\left(x_{0}, \theta_{i}\left(x, x_{0}\right)\right)>0, \quad i \in \mathcal{N}, \\
\left(d h_{j}\right)^{+}\left(x_{0}, \phi_{j}\left(x, x_{0}\right)\right)<0, \quad j \in J\left(x_{0}\right),
\end{gathered}
$$

has no solution $x \in X$.

Proof. Let $x_{0} \in X$ be a local weakly efficient solution for VFP and suppose there exists $\tilde{x} \in X$ such that inequalities (20)(22) are true.

For $i \in \mathcal{N}$, let $\varphi_{f_{i}}\left(x_{0}, \tilde{x}, \tau\right)=f_{i}\left(x_{0}+\tau \eta\left(\tilde{x}, x_{0}\right)\right)-f_{i}\left(x_{0}\right)$.

We observe that this function vanishes at $\tau=0$ and $\lim _{\tau \rightarrow 0^{+}} \tau^{-1}\left[\varphi_{f_{i}}\left(x_{0}, \tilde{x}, \tau\right)-\varphi_{f_{i}}\left(x_{0}, \tilde{x}, 0\right)\right]$ $=\lim _{\tau \rightarrow 0^{+}} \tau^{-1}\left[f_{i}\left(x_{0}+\tau \eta\left(\tilde{x}, x_{0}\right)\right)-f_{i}\left(x_{0}\right)\right]=$ $\left(d f_{i}\right)^{+}\left(x_{0}, \eta\left(\tilde{x}, x_{0}\right)\right) \leqq\left(d f_{i}\right)^{+}\left(x_{0}, \eta_{i}\left(\tilde{x}, x_{0}\right)\right) \quad<0$ using (17) and (20).

It follows that, for all $i \in \mathcal{N}, \varphi_{f_{i}}\left(x_{0}, \tilde{x}, \tau\right)<0$ if $\tau$ is in some open interval $\left(0, \delta_{f_{i}}\right), \delta_{f_{i}}>0$. Thus, for all $i \in \mathcal{N}$,

$$
f_{i}\left(x_{0}+\tau \eta\left(\tilde{x}, x_{0}\right)\right)<f_{i}\left(x_{0}\right), \quad \tau \in\left(0, \delta_{f_{i}}\right) .
$$

Similarly, by using (18) with (21) and (19) with (22), we get

$$
\begin{gathered}
g_{i}\left(x_{0}+\tau \eta\left(\tilde{x}, x_{0}\right)\right)>g_{i}\left(x_{0}\right), \quad \tau \in\left(0, \delta_{g_{i}}\right), \forall i \in \mathcal{N}, \\
h_{j}\left(x_{0}+\tau \eta\left(\tilde{x}, x_{0}\right)\right)<h_{j}\left(x_{0}\right)=0, \\
\tau \in\left(0, \delta_{h_{j}}\right), \forall j \in J\left(x_{0}\right),
\end{gathered}
$$

where, for all $i \in \mathcal{N}, \delta_{g_{i}}>0$ and, for all $j \in J\left(x_{0}\right), \delta_{h_{j}}>0$. 
Now, since, for $j \in \widetilde{J}\left(x_{0}\right), h_{j}\left(x_{0}\right)<0$ and $h_{j}$ is continuous at $x_{0}$, then there exists $\delta_{j}>0$ such that

$$
h_{j}\left(x_{0}+\tau \eta\left(\widetilde{x}, x_{0}\right)\right)<0, \quad \tau \in\left(0, \delta_{j}\right), \forall j \in \widetilde{J}\left(x_{0}\right) .
$$

Let $\delta_{0}=\min \left\{\delta_{f_{i}}, i \in \mathcal{N}, \delta_{g_{i}}, i \in \mathcal{N}, \delta_{h_{j}}, j \in J\left(x_{0}\right), \delta_{j}, j \in\right.$ $\left.\widetilde{J}\left(x_{0}\right)\right\}$. Then

$$
\left(x_{0}+\tau \eta\left(\tilde{x}, x_{0}\right)\right) \in N_{\delta_{0}}\left(x_{0}\right), \quad \tau \in\left(0, \delta_{0}\right) \text {, }
$$

where $N_{\delta_{0}}\left(x_{0}\right)$ is a neighborhood of $x_{0}$. Now, for all $\tau \in$ $\left(0, \delta_{0}\right)$, we have

$$
\begin{gathered}
f_{i}\left(x_{0}+\tau \eta\left(\tilde{x}, x_{0}\right)\right)<f_{i}\left(x_{0}\right), \quad i \in \mathcal{N}, \\
g_{i}\left(x_{0}+\tau \eta\left(\tilde{x}, x_{0}\right)\right)>g_{i}\left(x_{0}\right), \quad i \in \mathcal{N}, \\
h_{j}\left(x_{0}+\tau \eta\left(\tilde{x}, x_{0}\right)\right)<0, \quad j \in K .
\end{gathered}
$$

By (26) and (29), we get $\left(x_{0}+\tau \eta\left(\tilde{x}, x_{0}\right)\right) \in N_{\delta_{0}}\left(x_{0}\right) \cap X$, for all $\tau \in\left(0, \delta_{0}\right)$.

Using (27) and (28), for $R(x)=\left(f_{1}(x) / g_{1}(x), \ldots\right.$, $\left.f_{N}(x) / g_{N}(x)\right)$, we get

$$
R\left(x_{0}+\tau \eta\left(\tilde{x}, x_{0}\right)\right)<R\left(x_{0}\right),
$$

which contradicts the assumption that $x_{0}$ is a (local) weakly efficient solution for VFP. Hence, there exists no $x \in X$ satisfying the system (20)-(22). Thus the lemma is proved.

The following lemma given by Hayashi and Komiya [39] will be used.

Lemma 14. Let $S$ be a nonempty set in $\mathbb{R}^{n}$ and let $\psi: S \rightarrow \mathbb{R}^{m}$ be a convex-like function. Then either

$$
\psi(x)<0 \text { has a solution } x \in S
$$

or

$$
p^{T} \psi(x) \geqq 0 \text { for all } x \in S, \text { for some } p \in \mathbb{R}_{\geq}^{m},
$$

but both alternatives are never true (here the symbol $T$ denotes the transpose of matrix).

Preda [7], Mishra et al. [5], Niculescu [6], and Mishra and Rautela [4] have given necessary conditions for $x_{0} \in X$ to be a weakly efficient solution for VFP by taking the functions $f_{i}, g_{i}, i \in \mathcal{N}$, and $h_{j}, j \in J\left(x_{0}\right)$, semidifferentiable along the same direction $\eta\left(x, x_{0}\right)$. Now we give necessary efficiency criteria by considering each function $f_{i}, g_{i}, i \in \mathcal{N}$ (resp., $\left.h_{j}, j \in J\left(x_{0}\right)\right)$ semidifferentiable along its own direction $\eta_{i}\left(x, x_{0}\right), \theta_{i}\left(x, x_{0}\right), i \in \mathcal{N}\left(\right.$ resp., $\left.\phi_{j}\left(x, x_{0}\right), j \in J\left(x_{0}\right)\right)$.

In the next theorem, we obtain Fritz John type necessary efficiency conditions.

Theorem 15 (Fritz John type necessary efficiency conditions). Suppose that

(i) $x_{0}$ is a (local) weakly efficient solution for VFP; (ii) $h_{j}$ is continuous at $x_{0}$ for $j \in \widetilde{J}\left(x_{0}\right)$ and there exist vector functions $\eta_{i}: X \times D \rightarrow \mathbb{R}^{n}, \theta_{i}: X \times D \rightarrow$ $\mathbb{R}^{n}, i \in \mathcal{N}$, and $\phi_{j}: X \times D \rightarrow \mathbb{R}^{n}, j \in J\left(x_{0}\right)$, which satisfy at $x_{0}$ with respect to $\eta: X \times D \rightarrow \mathbb{R}^{n}$ inequalities (17)-(19);

(iii) for all $i \in \mathcal{N}, f_{i}, g_{i}$ (for all $j \in J\left(x_{0}\right), h_{j}$ ) is semidifferentiable at $x_{0}$ along the direction $\eta_{i}, \theta_{i}\left(\phi_{j}\right)$ and let $L(x)=\left[\left(d f_{i}\right)^{+}\left(x_{0}, \eta_{i}\left(x, x_{0}\right)\right)\right.$, $-\left(d g_{i}\right)^{+}\left(x_{0}, \theta_{i}\left(x, x_{0}\right)\right), i \in \mathcal{N},\left(d h_{j}\right)^{+}\left(x_{0}, \phi_{j}\left(x, x_{0}\right)\right)$, $\left.j \in J\left(x_{0}\right)\right] \in \mathbb{R}^{2 N+J}$ be a convex-like function of $x$ on $X$.

Then there exists $(\mu, \lambda, \delta) \in \mathbb{R}_{\geq}^{2 N+J}$ such that $\left(x_{0}, \mu, \lambda, \delta\right)$ satisfies

$$
\begin{array}{r}
\sum_{i=1}^{N} \mu_{i}\left(d f_{i}\right)^{+}\left(x_{0}, \eta_{i}\left(x, x_{0}\right)\right)-\sum_{i=1}^{N} \lambda_{i}\left(d g_{i}\right)^{+}\left(x_{0}, \theta_{i}\left(x, x_{0}\right)\right) \\
+\sum_{j \in J\left(x_{0}\right)} \delta_{j}\left(d h_{j}\right)^{+}\left(x_{0}, \phi_{j}\left(x, x_{0}\right)\right) \geqq 0, \quad \forall x \in X .
\end{array}
$$

Proof. If the conditions (i) and (ii) are satisfied, then, by Lemma 13, system (20)-(22) has no solution for $x \in X$. Since, by hypothesis (iii), $L(x)=\left[\left(d f_{i}\right)^{+}\left(x_{0}, \eta_{i}\left(x, x_{0}\right)\right)\right.$, $-\left(d g_{i}\right)^{+}\left(x_{0}, \theta_{i}\left(x, x_{0}\right)\right), i \in \mathcal{N},\left(d h_{j}\right)^{+}\left(x_{0}, \phi_{j}\left(x, x_{0}\right)\right), j \in$ $\left.J\left(x_{0}\right)\right] \in \mathbb{R}^{2 N+J}$ is a convex-like function of $x$ on $X$, therefore, by Lemma 14 , there exists $p=(\mu, \lambda, \delta) \in \mathbb{R}_{\geq}^{2 N+J}$ such that relation (33) is satisfied.

Remark 16. As particular case of Theorem 15, if $\eta_{i}=\theta_{i}=\eta$, $\forall i \in \mathcal{N}, \phi_{j}=\eta$, and $\forall j \in J\left(x_{0}\right)$ (i.e., if we consider that all of the functions $f_{i}, g_{i}, i \in \mathcal{N}$, and $h_{j}, j \in J\left(x_{0}\right)$, are semidifferentiable at $x_{0}$ along the same direction $\eta$ ), we obtain Theorem 14 of Preda [7], Lemma 5 of Mishra et al. [5], Lemma 5 of Niculescu [6], and Lemma 2.5 of Mishra and Rautela [4].

Now, we define a constraint qualification given as follows.

Definition 17. Let $x_{0}$ be a feasible point of VFP and let $\theta_{i}$ : $X \times X \rightarrow \mathbb{R}^{n}, i \in \mathcal{N}, \phi_{j}: X \times X \rightarrow \mathbb{R}^{n}, j \in J\left(x_{0}\right)$ be vector functions.

(i) The function $h$ is said to satisfy the semiconstraint qualification at $x_{0} \in X$ with respect to $\left(\phi_{j}\right)_{j \in J\left(x_{0}\right)}$, if there exist $\bar{x} \in X$ such that

$$
\left(d h_{j}\right)^{+}\left(x_{0}, \phi_{j}\left(\bar{x}, x_{0}\right)\right)<0, \quad \forall j \in J\left(x_{0}\right) .
$$

(ii) The function $h$ is said to satisfy the semiconstraint qualification at $x_{0} \in X$ with respect to $\left(\left(g_{i}\right)_{i \in \mathcal{N}},\left(\theta_{i}\right)_{i \in \mathcal{N}},\left(\phi_{j}\right)_{j \in J\left(x_{0}\right)}\right)$, if there exist $\bar{x} \in X$ such that

$$
\begin{gathered}
\left(d g_{i}\right)^{+}\left(x_{0}, \theta_{i}\left(\bar{x}, x_{0}\right)\right)>0, \quad \forall i \in \mathcal{N}, \\
\left(d h_{j}\right)^{+}\left(x_{0}, \phi_{j}\left(\bar{x}, x_{0}\right)\right)<0, \quad \forall j \in J\left(x_{0}\right) .
\end{gathered}
$$

To prove Karush-Kuhn-Tucker type necessary efficiency conditions for VFP, we need to prove the following result. 
Theorem 18. Suppose that

(i) $x_{0}$ is a (local) weakly efficient solution for the following problem:

$$
\begin{array}{ll}
\text { Minimize } & \left(\varphi_{1}(x), \ldots, \varphi_{N}(x)\right), \\
\text { subject to } & h_{j}(x) \leqq 0, \quad j=1,2, \ldots, k,
\end{array}
$$

where $\varphi=\left(\varphi_{1}, \ldots, \varphi_{N}\right): D \rightarrow \mathbb{R}^{N}$;

(ii) $h_{j}$ is continuous at $x_{0}$ for $j \in \widetilde{J}\left(x_{0}\right)$ and there exist vector functions $\eta_{i}: X \times D \rightarrow \mathbb{R}^{n}, i \in \mathcal{N}$, and $\phi_{j}: X \times D \rightarrow \mathbb{R}^{n}, j \in J\left(x_{0}\right)$, which satisfy at $x_{0}$ with respect to $\eta: X \times D \rightarrow \mathbb{R}^{n}$ the following inequalities:

$$
\begin{array}{r}
\left(d \varphi_{i}\right)^{+}\left(x_{0}, \eta\left(x, x_{0}\right)\right) \leqq\left(d \varphi_{i}\right)^{+}\left(x_{0}, \eta_{i}\left(x, x_{0}\right)\right), \\
\forall x \in X, \forall i \in \mathcal{N}, \\
\left(d h_{j}\right)^{+}\left(x_{0}, \eta\left(x, x_{0}\right)\right) \leqq\left(d h_{j}\right)^{+}\left(x_{0}, \phi_{j}\left(x, x_{0}\right)\right), \\
\forall x \in X, \forall j \in J\left(x_{0}\right) ;
\end{array}
$$

(iii) for all $i \in \mathcal{N}, \varphi_{i}$ (for all $j \in J\left(x_{0}\right), h_{j}$ ) is semidifferentiable at $x_{0}$ along the direction $\eta_{i}$ $\left(\phi_{j}\right)$ and let $L_{1}(x)=\left[\left(d \varphi_{i}\right)^{+}\left(x_{0}, \eta_{i}\left(x, x_{0}\right)\right), i \in\right.$ $\left.\mathcal{N},\left(d h_{j}\right)^{+}\left(x_{0}, \phi_{j}\left(x, x_{0}\right)\right), j \in J\left(x_{0}\right)\right] \in \mathbb{R}^{N+J}$ be a convex-like function of $x$ on $X$;

(iv) the function $h$ satisfies the semiconstraint qualification at $x_{0} \in X$ with respect to $\left(\phi_{j}\right)_{j \in J\left(x_{0}\right)}$.

Then there exist $\mu \in \mathbb{R}_{\geq}^{N}$ and $\delta \in \mathbb{R}_{\geqq}^{J}$ such that $\left(x_{0}, \mu, \delta\right)$ satisfies

$$
\begin{aligned}
& \sum_{i=1}^{N} \mu_{i}\left(d \varphi_{i}\right)^{+}\left(x_{0}, \eta_{i}\left(x, x_{0}\right)\right) \\
& \quad+\sum_{j \in J\left(x_{0}\right)} \delta_{j}\left(d h_{j}\right)^{+}\left(x_{0}, \phi_{j}\left(x, x_{0}\right)\right) \geqq 0, \quad \forall x \in X .
\end{aligned}
$$

Proof. In the same line as in the proof of Theorem 15, we prove that there exists $p=(\mu, \delta) \in \mathbb{R}_{\geq}^{N+J}$ such that relation (38) is satisfied. Now it is enough to prove that $\mu \neq 0$. We proceed by contradiction. If $\mu=0$, then $\delta \neq 0$ and (38) takes the following form:

$$
\sum_{j \in J\left(x_{0}\right)} \delta_{j}\left(d h_{j}\right)^{+}\left(x_{0}, \phi_{j}\left(x, x_{0}\right)\right) \geqq 0, \quad \forall x \in X,
$$

which contradicts semiconstraint qualification (34). Hence $\mu \neq 0$.

For each $\lambda=\left(\lambda_{1}, \ldots, \lambda_{N}\right) \in \mathbb{R}_{+}^{N}$, where $\mathbb{R}_{+}^{N}$ denotes the positive orthant of $\mathbb{R}^{N}$, we consider

$$
\begin{aligned}
\left(\mathrm{VFP}_{\lambda}\right) \text { Minimize } & \left(f_{1}(x)-\lambda_{1} g_{1}(x), \ldots,\right. \\
& \left.f_{N}(x)-\lambda_{N} g_{N}(x)\right), \\
\text { subject to } \quad & h_{j}(x) \leqq 0, j=1,2, \ldots, k .
\end{aligned}
$$

The following lemma can be proved without difficulty.
Lemma 19 (see [7]). If $x_{0}$ is a (local) weakly efficient solution for VFP, then $x_{0}$ is a (local) weakly efficient solution for $\left(V F P_{\lambda^{0}}\right)$, where $\lambda_{i}^{0}=f_{i}\left(x_{0}\right) / g_{i}\left(x_{0}\right), i \in \mathcal{N}$.

Using this lemma and Theorem 18, we can derive KarushKuhn-Tucker type necessary efficiency conditions for the problem VFP.

Theorem 20 (Karush-Kuhn-Tucker type necessary efficiency conditions). Suppose that

(i) $x_{0}$ is a (local) weakly efficient solution for VFP;

(ii) $h_{j}$ is continuous at $x_{0}$ for $j \in \widetilde{J}\left(x_{0}\right)$ and there exist vector functions $\eta_{i}: X \times D \rightarrow \mathbb{R}^{n}, i \in \mathcal{N}$, and $\phi_{j}: X \times D \rightarrow \mathbb{R}^{n}, j \in J\left(x_{0}\right)$, which satisfy at $x_{0}$ with respect to $\eta: X \times D \rightarrow \mathbb{R}^{n}$ inequalities (17) and (19) with

$$
\begin{array}{r}
\left(d g_{i}\right)^{+}\left(x_{0}, \eta\left(x, x_{0}\right)\right) \geqq\left(d g_{i}\right)^{+}\left(x_{0}, \eta_{i}\left(x, x_{0}\right)\right), \\
\forall x \in X, \forall i \in \mathcal{N} ;
\end{array}
$$

(iii) for all $i \in \mathcal{N}, f_{i}, g_{i}$ (for all $\left.j \in J\left(x_{0}\right), h_{j}\right)$ is semidifferentiable at $x_{0}$ along the direction $\eta_{i}\left(\phi_{j}\right)$ and let $L_{2}(x)=$ $\left[\left[\left(d f_{i}\right)^{+}\left(x_{0}, \eta_{i}\left(x, x_{0}\right)\right)-\lambda_{j}^{0}\left(d g_{i}\right)^{+}\left(x_{0}, \eta_{i}\left(x, x_{0}\right)\right)\right], i \in\right.$ $\left.\mathcal{N},\left(d h_{j}\right)^{+}\left(x_{0}, \phi_{j}\left(x, x_{0}\right)\right), j \in J\left(x_{0}\right)\right] \in \mathbb{R}^{N+J}$ be a convex-like function of $x$ on $X$, where $\lambda^{0}=$ $\left(\lambda_{1}^{0}, \ldots, \lambda_{N}^{0}\right), \lambda_{i}^{0}=f_{i}\left(x_{0}\right) / g_{i}\left(x_{0}\right), i \in \mathcal{N} ;$

(iv) the function $h$ satisfies the semiconstraint qualification at $x_{0} \in X$ with respect to $\left(\phi_{j}\right)_{j \in J\left(x_{0}\right)}$.

Then there exist $\mu \in \mathbb{R}_{\geq}^{N}$ and $\delta \in \mathbb{R}_{\geq}^{J}$ such that $\left(x_{0}, \mu, \lambda^{0}, \delta\right)$ satisfies

$$
\begin{array}{r}
\sum_{i=1}^{N} \mu_{i}\left[\left(d f_{i}\right)^{+}\left(x_{0}, \eta_{i}\left(x, x_{0}\right)\right)-\lambda_{i}^{0}\left(d g_{i}\right)^{+}\left(x_{0}, \eta_{i}\left(x, x_{0}\right)\right)\right] \\
+\sum_{j \in J\left(x_{0}\right)} \delta_{j}\left(d h_{j}\right)^{+}\left(x_{0}, \phi_{j}\left(x, x_{0}\right)\right) \geqq 0, \quad \forall x \in X .
\end{array}
$$

Proof. Let $x_{0}$ be a (local) weakly efficient solution for VFP. According to Lemma 19 we have that $x_{0}$ is a (local) weakly efficient solution for $\left(\operatorname{VFP}_{\lambda^{0}}\right)$. Now applying Theorem 18 to problem $\left(\operatorname{VFP}_{\lambda^{0}}\right)$, we get that there exist $\mu \in \mathbb{R}_{\geq}^{N}$ and $\delta \in \mathbb{R}_{\geq}^{J}$ such that relation (42) is satisfied, and the theorem is proved.

In the Karush-Kuhn-Tucker type necessary efficiency condition (42) of Theorem 20, the functions $f_{i}$ and $g_{i}$ are considered semidifferentiable at $x_{0}$ along the same direction $\eta_{i}, i \in \mathcal{N}$. To obtain a necessary condition with different directions $\eta_{i}$ and $\theta_{i}$, we need to use the second variant of the semiconstraint qualification given in Definition 17.

Theorem 21 (Karush-Kuhn-Tucker type necessary efficiency conditions). Suppose that the hypotheses (i), (ii), and (iii) of Theorem 15 are satisfied and the function $h$ satisfies the 
semiconstraint qualification at $x_{0} \in X$ with respect to $\left(\left(g_{i}\right)_{i \in \mathcal{N}},\left(\theta_{i}\right)_{i \in \mathcal{N}},\left(\phi_{j}\right)_{j \in J\left(x_{0}\right)}\right)$. Then there exist $\mu \in \mathbb{R}_{\geq}^{N}, \lambda \in$ $\mathbb{R}_{\geqq}^{N}$, and $\delta \in \mathbb{R}_{\geqq}^{J}$ such that $\left(x_{0}, \mu, \lambda, \delta\right)$ satisfies relation (33).

Proof. Based on Theorem 15, we obtain the existence of $(\mu, \lambda, \delta) \in \mathbb{R}_{\geq}^{2 N+J}$ such that $\left(x_{0}, \mu, \lambda, \delta\right)$ satisfies relation (33). Now it is enough to prove that $\mu \neq 0$. We proceed by contradiction. If $\mu=0$, then $(\lambda, \delta) \neq 0$ and (33) takes the following form:

$$
\begin{aligned}
& -\sum_{i=1}^{N} \lambda_{i}\left(d g_{i}\right)^{+}\left(x_{0}, \theta_{i}\left(x, x_{0}\right)\right) \\
& \quad+\sum_{j \in J\left(x_{0}\right)} \delta_{j}\left(d h_{j}\right)^{+}\left(x_{0}, \phi_{j}\left(x, x_{0}\right)\right) \geqq 0, \quad \forall x \in X,
\end{aligned}
$$

which contradicts the semiconstraint qualification (ii) of Definition 17. Hence $\mu \neq 0$.

\section{Sufficient Efficiency Criteria}

In this section, we present some Karush-Kuhn-Tucker type sufficient efficiency conditions for a feasible solution to be efficient or weakly efficient for VFP under various types of generalized semilocally $\mathrm{V}$-type I-preinvex assumptions.

Theorem 22. Let $x_{0} \in X$ and suppose that there exist $(2 N+J)$ vector functions $\eta_{i}: X \times D \rightarrow \mathbb{R}^{n}, \theta_{i}: X \times D \rightarrow \mathbb{R}^{n}, i \in \mathcal{N}$ and $\phi_{j}: X \times D \rightarrow \mathbb{R}^{n}, j \in J\left(x_{0}\right)$, such that VFP is semilocally $V$-type I-preinvex at $x_{0}$ with respect to $\left(\eta_{i}\right)_{i \in \mathscr{N}},\left(\theta_{i}\right)_{i \in \mathcal{N}}$, and $\left(\phi_{j}\right)_{j \in J\left(x_{0}\right)}$. If there exist vectors $\mu \in \mathbb{R}_{\geq}^{N}, \lambda \in \mathbb{R}_{\geqq}^{N}$, and $\delta \in \mathbb{R}_{\geqq}^{J}$ such that

$$
\begin{aligned}
& \sum_{i=1}^{N} \mu_{i}\left(d f_{i}\right)^{+}\left(x_{0}, \eta_{i}\left(x, x_{0}\right)\right) \\
& \quad+\sum_{j \in J\left(x_{0}\right)} \delta_{j}\left(d h_{j}\right)^{+}\left(x_{0}, \phi_{j}\left(x, x_{0}\right)\right) \geqq 0, \quad \forall x \in X, \\
& \quad\left(d g_{i}\right)^{+}\left(x_{0}, \theta_{i}\left(x, x_{0}\right)\right) \leqq 0, \quad \forall x \in X, \forall i \in \mathcal{N},
\end{aligned}
$$

then $x_{0}$ is a weakly efficient solution for VFP.

Proof. Suppose that $x_{0}$ is not a weakly efficient solution of VFP. Then there exists a feasible solution $\bar{x} \in X$ of VFP such that

$$
\frac{f_{i}(\bar{x})}{g_{i}(\bar{x})}<\frac{f_{i}\left(x_{0}\right)}{g_{i}\left(x_{0}\right)}, \quad \forall i \in \mathcal{N} .
$$

Since VFP is semilocally V-type I-preinvex at $x_{0}$ with respect to $\left(\eta_{i}\right)_{i \in \mathcal{N}},\left(\theta_{i}\right)_{i \in \mathcal{N}}$, and $\left(\phi_{j}\right)_{j \in J\left(x_{0}\right)}$, we get that inequalities $(6)$, (7), and (8) are true for $x=\bar{x}$.
Multiplying (6) by $\mu_{i}, i \in \mathcal{N}$, and (8) by $\delta_{j}, j \in J\left(x_{0}\right)$, then summing the obtained relation and using (44), we get

$$
\begin{aligned}
\sum_{i=1}^{N} \mu_{i}\left[f_{i}(\bar{x})-f_{i}\left(x_{0}\right)\right]-\sum_{j \in J\left(x_{0}\right)} \delta_{j} h_{j}\left(x_{0}\right) \\
\geqq \sum_{i=1}^{N} \mu_{i}\left(d f_{i}\right)^{+}\left(x_{0}, \eta_{i}\left(\bar{x}, x_{0}\right)\right) \\
\quad+\sum_{j \in J\left(x_{0}\right)} \delta_{j}\left(d h_{j}\right)^{+}\left(x_{0}, \phi_{j}\left(\bar{x}, x_{0}\right)\right) \geqq 0 .
\end{aligned}
$$

From the above inequality and the fact that $h_{j}\left(x_{0}\right)=0, \forall j \in$ $J\left(x_{0}\right)$, it follows that

$$
\sum_{i=1}^{N} \mu_{i}\left[f_{i}(\bar{x})-f_{i}\left(x_{0}\right)\right] \geqq 0 .
$$

Since $\mu \geq 0$, from (48) we obtain that there exists $i_{0} \in \mathcal{N}$ such that

$$
f_{i_{0}}(\bar{x}) \geqq f_{i_{0}}\left(x_{0}\right) .
$$

By (45) and (7) it follows that

$$
g_{i}(\bar{x}) \leqq g_{i}\left(x_{0}\right), \quad \forall i \in \mathcal{N}
$$

Now using (49), (50), and $f \geqq 0, g>0$, we obtain

$$
\frac{f_{i_{0}}(\bar{x})}{g_{i_{0}}(\bar{x})} \geqq \frac{f_{i_{0}}\left(x_{0}\right)}{g_{i_{0}}\left(x_{0}\right)},
$$

which is a contradiction to (46). Thus $x_{0}$ is a weakly efficient solution for VFP and the theorem is proved.

Remark 23. As particular case of Theorem 22, if $\eta_{i}=\theta_{i}=$ $\eta, \forall i \in \mathcal{N}$, and $\phi_{j}=\eta, \forall j \in J\left(x_{0}\right)$, we obtain Theorem 1 of Mishra et al. [5].

Theorem 24. Let $x_{0} \in X$ and suppose that there exist $(2 N+J)$ vector functions $\eta_{i}: X \times D \rightarrow \mathbb{R}^{n}, \theta_{i}: X \times D \rightarrow \mathbb{R}^{n}, i \in \mathcal{N}$, and $\phi_{j}: X \times D \rightarrow \mathbb{R}^{n}, j \in J\left(x_{0}\right)$, such that VFP is semilocally $V$-type I-preinvex at $x_{0}$ with respect to $\left(\eta_{i}\right)_{i \in \mathcal{N}},\left(\theta_{i}\right)_{i \in \mathcal{N}}$, and $\left(\phi_{j}\right)_{j \in J\left(x_{0}\right)}$. If there exist vectors $\mu \in \mathbb{R}_{\geq}^{N}, \lambda \in \mathbb{R}_{\geqq}^{N}\left(\lambda_{i}=\right.$ $\left.f_{i}\left(x_{0}\right) / g_{i}\left(x_{0}\right), i \in \mathcal{N}\right)$, and $\delta \in \mathbb{R}_{\geqq}^{J}$ such that

$$
\begin{aligned}
& \sum_{i=1}^{N} \mu_{i}\left[\left(d f_{i}\right)^{+}\left(x_{0}, \eta_{i}\left(x, x_{0}\right)\right)\right. \\
& \left.\quad-\lambda_{i}\left(d g_{i}\right)^{+}\left(x_{0}, \theta_{i}\left(x, x_{0}\right)\right)\right] \\
& \quad+\sum_{j \in J\left(x_{0}\right)} \delta_{j}\left(d h_{j}\right)^{+}\left(x_{0}, \phi_{j}\left(x, x_{0}\right)\right) \geqq 0, \quad \forall x \in X,
\end{aligned}
$$

then $x_{0}$ is a weakly efficient solution for VFP. 
Proof. Suppose that $x_{0}$ is not a weakly efficient solution of VFP. Then there exists a feasible solution $\bar{x} \in X$ of VFP such that

$$
\frac{f_{i}(\bar{x})}{g_{i}(\bar{x})}<\frac{f_{i}\left(x_{0}\right)}{g_{i}\left(x_{0}\right)}, \quad \forall i \in \mathcal{N},
$$

$$
\text { that is, } f_{i}(\bar{x})<\lambda_{i} g_{i}(\bar{x}), \quad \forall i \in \mathcal{N} \text {. }
$$

Since VFP is semilocally V-type I-preinvex at $x_{0}$ with respect to $\left(\eta_{i}\right)_{i \in \mathcal{N}},\left(\theta_{i}\right)_{i \in \mathcal{N}}$, and $\left(\phi_{j}\right)_{j \in J\left(x_{0}\right)}$, we get that inequalities (6), (7), and (8) are true for $x=\bar{x}$.

Using these inequalities, $\mu \geq 0, \lambda \geqq 0, \delta \geqq 0$, and (52), we get

$$
\begin{gathered}
\sum_{i=1}^{N} \mu_{i}\left[f_{i}(\bar{x})-f_{i}\left(x_{0}\right)\right]-\sum_{i=1}^{N} \mu_{i} \lambda_{i}\left[g_{i}(\bar{x})-g_{i}\left(x_{0}\right)\right] \\
\quad-\sum_{j \in J\left(x_{0}\right)} \delta_{j} h_{j}\left(x_{0}\right) \\
\geqq \sum_{i=1}^{N} \mu_{i}\left[\left(d f_{i}\right)^{+}\left(x_{0}, \eta_{i}\left(\bar{x}, x_{0}\right)\right)\right. \\
\left.-\lambda_{i}\left(d g_{i}\right)^{+}\left(x_{0}, \theta_{i}\left(\bar{x}, x_{0}\right)\right)\right] \\
+\sum_{j \in J\left(x_{0}\right)} \delta_{j}\left(d h_{j}\right)^{+}\left(x_{0}, \phi_{j}\left(\bar{x}, x_{0}\right)\right) \geqq 0 .
\end{gathered}
$$

Therefore,

$$
\begin{aligned}
\sum_{i=1}^{N} \mu_{i} & {\left[\left(f_{i}(\bar{x})-\lambda_{i} g_{i}(\bar{x})\right)-\left(f_{i}\left(x_{0}\right)-\lambda_{i} g_{i}\left(x_{0}\right)\right)\right] } \\
& -\sum_{j \in J\left(x_{0}\right)} \delta_{j} h_{j}\left(x_{0}\right) \geqq 0 .
\end{aligned}
$$

Since $\lambda_{i}=f_{i}\left(x_{0}\right) / g_{i}\left(x_{0}\right), i \in \mathcal{N}$, and $h_{j}\left(x_{0}\right)=0, \forall j \in J\left(x_{0}\right)$, we obtain

$$
\sum_{i=1}^{N} \mu_{i}\left(f_{i}(\bar{x})-\lambda_{i} g_{i}(\bar{x})\right) \geqq 0 .
$$

Since $\mu \geq 0$, from (57) we obtain that there exists $i_{0} \in \mathcal{N}$ such that

$$
f_{i_{0}}(\bar{x})-\lambda_{i_{0}} g_{i_{0}}(\bar{x}) \geqq 0,
$$

that is,

$$
\frac{f_{i_{0}}(\bar{x})}{g_{i_{0}}(\bar{x})} \geqq \frac{f_{i_{0}}\left(x_{0}\right)}{g_{i_{0}}\left(x_{0}\right)},
$$

which is a contradiction to (53). Thus $x_{0}$ is a weakly efficient solution for VFP and the theorem is proved.

Remark 25. It is easy to see that, in Theorem 24, if the problem VFP is semistrictly semilocally V-type I-preinvex at $x_{0}$ with respect to $\left(\eta_{i}\right)_{i \in \mathcal{N}},\left(\theta_{i}\right)_{i \in \mathcal{N}}$, and $\left(\phi_{j}\right)_{j \in J\left(x_{0}\right)}$ (instead of semilocally V-type I-preinvex), the point $x_{0}$ will be efficient for VFP.
Remark 26. As a particular case of Theorem 24, if $\eta_{i}=\theta_{i}=$ $\eta, \forall i \in \mathcal{N}$, and $\phi_{j}=\eta, \forall j \in J\left(x_{0}\right)$, we obtain Theorem 2 of Mishra et al. [5].

Preda [7], Mishra et al. [5], Niculescu [6], and Mishra and Rautela [4] have given sufficient efficiency conditions for a feasible solution to be weakly efficient under various types of (generalized) $\eta$-semilocally (type I-) preinvex (univex) assumptions. In the following theorem, we give sufficient efficiency conditions for a feasible solution to be efficient involving generalized semilocally V-type I-preinvex functions.

Theorem 27. Let $x_{0} \in X$ and suppose that there exist $(2 N+J)$ vector functions $\eta_{i}: X \times D \rightarrow \mathbb{R}^{n}, \theta_{i}: X \times D \rightarrow$ $\mathbb{R}^{n}, i \in \mathcal{N}, \phi_{j}: X \times D \rightarrow \mathbb{R}^{n}, j \in J\left(x_{0}\right)$, and vectors $\mu \in \mathbb{R}_{\geq}^{N}, \lambda \in \mathbb{R}_{\geqq}^{N}\left(\lambda_{i}=f_{i}\left(x_{0}\right) / g_{i}\left(x_{0}\right), i \in \mathcal{N}\right)$, and $\delta \in \mathbb{R}_{\geqq}^{J}$ such that relation (52) is satisfied. If any one of the following conditions holds,

(a) VFP is semilocally strictly pseudo quasi-V-type Ipreinvex at $x_{0}$ with respect to $\left(\eta_{i}\right)_{i \in \mathscr{N}},\left(\theta_{i}\right)_{i \in \mathcal{N}}$, and $\left(\phi_{j}\right)_{j \in J\left(x_{0}\right)}$ and for $\mu, \lambda$, and $\delta$;

(b) VFP is semilocally quasi strictly pseudo-V-type Ipreinvex at $x_{0}$ with respect to $\left(\eta_{i}\right)_{i \in \mathcal{N}},\left(\theta_{i}\right)_{i \in \mathcal{N}}$, and $\left(\phi_{j}\right)_{j \in J\left(x_{0}\right)}$ and for $\mu, \lambda$, and $\delta$,

then $x_{0}$ is an efficient solution for VFP.

Proof. Suppose that $x_{0}$ is not an efficient solution of VFP. Then there exists a feasible solution $\bar{x} \in X$ of VFP such that for some $p \in \mathcal{N}$

$$
\begin{gathered}
\frac{f_{p}(\bar{x})}{g_{p}(\bar{x})}<\frac{f_{p}\left(x_{0}\right)}{g_{p}\left(x_{0}\right)}, \quad \frac{f_{i}(\bar{x})}{g_{i}(\bar{x})} \leqq \frac{f_{i}\left(x_{0}\right)}{g_{i}\left(x_{0}\right)}, \quad \forall i \in \mathcal{N}, i \neq p, \\
\text { that is, } f_{p}(\bar{x})<\lambda_{p} g_{p}(\bar{x}), \quad f_{i}(\bar{x}) \leqq \lambda_{i} g_{i}(\bar{x}), \\
\forall i \in \mathcal{N}, \quad i \neq p,
\end{gathered}
$$

which is equivalent to

$$
\begin{array}{r}
f_{p}(\bar{x})-\lambda_{p} g_{p}(\bar{x})<f_{p}\left(x_{0}\right)-\lambda_{p} g_{p}\left(x_{0}\right), \\
f_{i}(\bar{x})-\lambda_{i} g_{i}(\bar{x}) \leqq f_{i}\left(x_{0}\right)-\lambda_{i} g_{i}\left(x_{0}\right), \\
\forall i \in \mathcal{N}, i \neq p .
\end{array}
$$

Since $\mu \geq 0$, from (61) and (62) we obtain

$$
\sum_{i=1}^{N} \mu_{i}\left[f_{i}(\bar{x})-\lambda_{i} g_{i}(\bar{x})\right] \leqq \sum_{i=1}^{N} \mu_{i}\left[f_{i}\left(x_{0}\right)-\lambda_{i} g_{i}\left(x_{0}\right)\right]
$$

With condition (a), by using the reverse implication in (9) (in view of Definition 7), we deduce that

$$
\sum_{i=1}^{N} \mu_{i}\left[\left(d f_{i}\right)^{+}\left(x_{0}, \eta_{i}\left(\bar{x}, x_{0}\right)\right)-\lambda_{i}\left(d g_{i}\right)^{+}\left(x_{0}, \theta_{i}\left(\bar{x}, x_{0}\right)\right)\right]<0 .
$$


From $h_{j}\left(x_{0}\right)=0, \delta_{j} \geqq 0, \forall j \in J\left(x_{0}\right)$ (in view of Definition 7), we get

$$
\sum_{j \in J\left(x_{0}\right)} \delta_{j}\left(d h_{j}\right)^{+}\left(x_{0}, \phi_{j}\left(\bar{x}, x_{0}\right)\right) \leqq 0 .
$$

Now, by (64) and (65), we obtain

$$
\begin{aligned}
& \sum_{i=1}^{N} \mu_{i} {\left[\left(d f_{i}\right)^{+}\left(x_{0}, \eta_{i}\left(\bar{x}, x_{0}\right)\right)-\lambda_{i}\left(d g_{i}\right)^{+}\left(x_{0}, \theta_{i}\left(\bar{x}, x_{0}\right)\right)\right] } \\
&+\sum_{j \in J\left(x_{0}\right)} \delta_{j}\left(d h_{j}\right)^{+}\left(x_{0}, \phi_{j}\left(\bar{x}, x_{0}\right)\right)<0,
\end{aligned}
$$

which is a contradiction to (52).

The proof of part (b) is very similar to the proof of part (a), except that, for this case, by using the implication in (11) in view of Definition 8 , inequality (64) becomes not strict (ฏ) and, by using the reverse implication in (12), inequality (65) becomes strict $(<)$. Thus we get the contradiction again, and the theorem will be proved.

Remark 28. In Theorems 24 and 27, we do not need to require that $f \geqq 0$. Thus, in Example 29 that uses Theorem 27, it is sufficient to have $g>0$.

In order to illustrate the obtained results, we will give an example of multiobjective fractional optimization problem in which the efficient solution will be obtained by the application of Theorem 27, whereas it will be impossible to apply for this purpose the sufficient efficiency conditions devoted to locally Lipschitz functions or to $\eta$-semidifferentiable functions (with the same $\eta$ ). In particular, the sufficient efficiency conditions given in Mishra and Rautela [4], Mishra et al. [5], Niculescu [6], and Preda [7] are not applicable.

Example 29. We consider the following multiobjective fractional optimization problem:

$$
\begin{array}{ll}
\text { Minimize } & \frac{f(x)}{g(x)}=\left(\frac{f_{1}(x)}{g_{1}(x)}, \frac{f_{2}(x)}{g_{2}(x)}, \frac{f_{3}(x)}{g_{3}(x)}\right), \\
\text { subject to } & h(x) \leqq 0,
\end{array}
$$

where $D=(]-\sqrt{2}, \sqrt{2}[)^{2} \subset \mathbb{R}^{2}, f, g: D \rightarrow \mathbb{R}^{3}$, and $h:$ $D \rightarrow \mathbb{R}$ defined by

$$
\begin{gathered}
f_{1}\left(x_{1}, x_{2}\right)= \begin{cases}0, & \text { if } x_{1}=0 \text { or } x_{2}=0 ; \\
-1+x_{1}^{2}, & \text { otherwise, }\end{cases} \\
f_{2}\left(x_{1}, x_{2}\right)= \begin{cases}-1+x_{1}^{3}, & \text { if } x_{1} \neq 0 \text { and } x_{2}=0 ; \\
-1+x_{2}^{4}, & \text { if } x_{1}=0 \text { and } x_{2} \neq 0 ; \\
0, & \text { otherwise, }\end{cases} \\
f_{3}\left(x_{1}, x_{2}\right)= \begin{cases}2, & \text { if } x_{1}=0 \text { and } x_{2}=0 ; \\
2+x_{1}^{2}+x_{2}^{2}, & \text { otherwise, }\end{cases} \\
g_{1}\left(x_{1}, x_{2}\right)= \begin{cases}1, & \text { if } x_{1}=0 \text { or } x_{2}=0 ; \\
2-x_{2}^{2}, & \text { otherwise, }\end{cases}
\end{gathered}
$$

$$
\begin{gathered}
g_{2}\left(x_{1}, x_{2}\right)= \begin{cases}2-x_{1}^{2}, & \text { if } x_{1} \neq 0 \text { and } x_{2}=0 ; \\
2-x_{2}^{2}, & \text { if } x_{1}=0 \text { and } x_{2} \neq 0 ; \\
1, & \text { otherwise, }\end{cases} \\
g_{3}\left(x_{1}, x_{2}\right)=1, \quad \forall x \in \mathbb{R}^{2}, \\
h\left(x_{1}, x_{2}\right)= \begin{cases}0, & \text { if } x_{1}=0 \text { or } x_{2}=0 ; \\
-1+x_{2}^{2}, & \text { otherwise. }\end{cases}
\end{gathered}
$$

The set $X$ of feasible solutions of problem is nonempty. Observe that $f_{1}, f_{2}, g_{1}, g_{2}$, and $h$ are not continuous at $x_{0}=$ $(0,0) \in X$, and, consequently, they are not locally Lipschitz at this point. In addition, clearly, the functions $f_{1}, f_{2}, g_{1}, g_{2}$, and $h$ are not differentiable at $x_{0}$ but only semidifferentiable functions at that point.

There exists no function $\eta: \mathbb{R}^{2} \times \mathbb{R}^{2} \rightarrow \mathbb{R}^{2}, \eta \neq \equiv(0,0)$, $\mu \in \mathbb{R}_{\geq}^{3}, \lambda \in \mathbb{R}_{\geqq}^{3}$, and $\delta \in \mathbb{R}_{\geqq}$such that

$$
\begin{aligned}
& \sum_{i=1}^{3} \mu_{i} {\left[\left(d f_{i}\right)^{+}\left(x_{0}, \eta\left(x, x_{0}\right)\right)-\lambda_{i}\left(d g_{i}\right)^{+}\left(x_{0}, \eta\left(x, x_{0}\right)\right)\right] } \\
&+\delta(d h)^{+}\left(x_{0}, \eta\left(x, x_{0}\right)\right) \geqq 0, \quad \forall x \in X .
\end{aligned}
$$

Then, the sufficient efficiency conditions given in Mishra and Rautela [4], Mishra et al. [5], Niculescu [6], and Preda [7] are not applicable. However, there exist vector functions $\eta_{1}\left(x, x_{0}\right)=\left(1+x_{1}^{2}, 0\right), \quad \eta_{2}\left(x, x_{0}\right)=\left(1+x_{1}^{2}, 1+x_{2}^{2}\right)$, $\eta_{3}\left(x, x_{0}\right)=\left(x_{1}, x_{2}\right), \theta_{1}\left(x, x_{0}\right)=\left(1+x_{2}^{2}, 0\right), \theta_{2}\left(x, x_{0}\right)=$ $\left(1+x_{2}^{2}, 1+x_{1}^{2}\right), \theta_{3}\left(x, x_{0}\right)=\left(x_{2}, x_{1}\right), \phi\left(x, x_{0}\right)=(0,1+$ $x_{2}^{2}$ ) and scalars $\mu_{1}=\mu_{2}=0, \mu_{3}=1, \lambda_{1}=\lambda_{2}=$ $0, \lambda_{3}=2, \delta=1$ such that relation (52) is satisfied and problem (67) is semilocally strictly pseudo quasi-V-type Ipreinvex at $x_{0}$ with respect to $\left(\eta_{i}\right)_{i=1,2,3},\left(\theta_{i}\right)_{i=1,2,3}$, and $\phi$ and for $\mu=\left(\mu_{1}, \mu_{2}, \mu_{3}\right), \lambda=\left(\lambda_{1}, \lambda_{2}, \lambda_{3}\right)$, and $\delta$. It follows that, by Theorem 27, $x_{0}$ is an efficient solution for the given multiobjective fractional optimization problem.

\section{General Mond-Weir Type Duality}

We associate, for VFP, a general Mond-Weir dual GMWD given as follows:

$$
\begin{gathered}
\text { (GMWD) Maximize } \psi\left(y, \mu, \lambda, \delta,\left(\eta_{i}\right)_{i \in \mathcal{N}},\left(\phi_{j}\right)_{j \in K}\right) \\
=\lambda=\left(\lambda_{1}, \ldots, \lambda_{N}\right),
\end{gathered}
$$

subject to

$$
\begin{gathered}
\sum_{i=1}^{N} \mu_{i}\left[\left(d f_{i}\right)^{+}\left(y, \eta_{i}(x, y)\right)-\lambda_{i}\left(d g_{i}\right)^{+}\left(y, \eta_{i}(x, y)\right)\right] \\
+\sum_{j=1}^{k} \delta_{j}\left(d h_{j}\right)^{+}\left(y, \phi_{j}(x, y)\right) \geqq 0, \quad \forall x \in X, \\
f_{i}(y)-\lambda_{i} g_{i}(y)+\delta_{J_{0}}^{T} h_{J_{0}}(y) \geqq 0, \quad i \in \mathcal{N},
\end{gathered}
$$




$$
\begin{gathered}
\delta_{J_{s}}^{T} h_{J_{s}}(y) \geqq 0, \quad s=1,2, \ldots, \alpha, \\
\mu^{T} e=1, \quad \mu \in \mathbb{R}_{\geq}^{N}, \\
\lambda \in \mathbb{R}_{\geqq}^{N}, \quad \delta \in \mathbb{R}_{\geqq}^{k}, \quad y \in D, \\
\eta_{i}: X \times D \longrightarrow \mathbb{R}^{n}, \quad i \in \mathcal{N}, \\
\phi_{j}: X \times D \longrightarrow \mathbb{R}^{n}, \quad j \in K,
\end{gathered}
$$

where $\alpha \geqq 1, J_{s} \cap J_{t}=\emptyset$ for $s \neq t$ and $\bigcup_{s=0}^{\alpha} J_{s}=K$. Here $\delta_{J_{s}}=\left(\delta_{j}\right)_{j \in J_{s}}, h_{J_{s}}=\left(h_{j}\right)_{j \in J_{s}}$. Let $Y$ be the set of all feasible solutions of problem GMWD.

Now, we establish certain duality results between VFP and GMWD by considering that, for all $i \in \mathcal{N}, f_{i}, g_{i}$ (for all $j \in$ $\left.J\left(x_{0}\right), h_{j}\right)$ is semidifferentiable on $D$ along its own direction $\eta_{i}\left(\phi_{j}\right)$ instead of the same direction $\eta$.

Theorem 30 (weak duality). Assume that, for all feasible $x$ for VFP and all feasible $\left(y, \mu, \lambda, \delta,\left(\eta_{i}\right)_{i \in \mathscr{N}},\left(\phi_{j}\right)_{j \in K}\right)$ for GMWD, any of the following holds:

(a) the problem VFP is semilocally extendedly pseudo partially quasi-V-type I-preinvex at $y$ with respect to $\left\{\left(\eta_{i}\right)_{i \in \mathcal{N}},\left(\phi_{j}\right)_{j \in J_{0}}\right\}$ and $\left\{\left(\phi_{j}\right)_{j \in J_{s}}, s=1,2, \ldots, \alpha\right\}$ and for $\mu, \lambda$, and $\delta$ with $\mu>0$;

(b) the problem VFP is semilocally strictly extendedly pseudo partially quasi-V-type I-preinvex at $y$ with respect to $\left\{\left(\eta_{i}\right)_{i \in \mathcal{N}}, \quad\left(\phi_{j}\right)_{j \in J_{0}}\right\}$ and $\left\{\left(\phi_{j}\right)_{j \in J_{s}}, s=\right.$ $1,2, \ldots, \alpha\}$ and for $\mu, \lambda$, and $\delta$;

(c) the problem VFP is semilocally extendedly quasi strictly partially pseudo-V-type I-preinvex at $y$ with respect to $\left\{\left(\eta_{i}\right)_{i \in \mathscr{N}},\left(\phi_{j}\right)_{j \in J_{0}}\right\}$ and $\left\{\left(\phi_{j}\right)_{j \in J_{s}}, s=1,2, \ldots, \alpha\right\}$ and for $\mu, \lambda$, and $\delta$.

Then the following cannot hold:

$$
\begin{gathered}
f_{i}(x)-\lambda_{i} g_{i}(x) \leqq 0 \text { foreach } i \in \mathcal{N} \\
f_{i_{0}}(x)-\lambda_{i_{0}} g_{i_{0}}(x)<0 \text { forsome } i_{0} \in \mathcal{N} .
\end{gathered}
$$

Proof. By condition (a), since the problem VFP is semilocally extendedly pseudo partially quasi-V-type I-preinvex at $y$ with respect to $\left\{\left(\eta_{i}\right)_{i \in \mathcal{N}},\left(\phi_{j}\right)_{j \in J_{0}}\right\}$ and $\left\{\left(\phi_{j}\right)_{j \in J_{s}}, s=1,2, \ldots, \alpha\right\}$ and for $\mu, \lambda$, and $\delta$, then

$$
\begin{gathered}
\sum_{i=1}^{N} \mu_{i}\left[\left(d f_{i}\right)^{+}\left(y, \eta_{i}(x, y)\right)-\lambda_{i}\left(d g_{i}\right)^{+}\left(y, \eta_{i}(x, y)\right)\right] \\
+\sum_{j \in J_{0}} \delta_{j}\left(d h_{j}\right)^{+}\left(y, \phi_{j}(x, y)\right) \geqq 0 \\
\Longrightarrow \sum_{i=1}^{N} \mu_{i}\left[f_{i}(x)-\lambda_{i} g_{i}(x)\right]+\sum_{j \in J_{0}} \delta_{j} h_{j}(x) \\
\geqq \sum_{i=1}^{N} \mu_{i}\left[f_{i}(y)-\lambda_{i} g_{i}(y)\right]+\sum_{j \in J_{0}} \delta_{j} h_{j}(y),
\end{gathered}
$$

$$
\begin{array}{r}
\sum_{j \in J_{s}} \delta_{j} h_{j}(y) \geqq 0 \Longrightarrow \sum_{j \in J_{s}} \delta_{j}\left(d h_{j}\right)^{+}\left(y, \phi_{j}(x, y)\right) \leqq 0, \\
s=1,2, \ldots, \alpha .
\end{array}
$$

From (73) and (80), we get

$$
\sum_{j \in J_{s}} \delta_{j}\left(d h_{j}\right)^{+}\left(y, \phi_{j}(x, y)\right) \leqq 0, \quad s=1,2, \ldots, \alpha
$$

Now we suppose contrary to the result of the theorem that (77) and (78) hold. Hence if (77) and (78) hold for some feasible $x$ for VFP and $\left(y, \mu, \lambda, \delta,\left(\eta_{i}\right)_{i \in \mathcal{N}},\left(\phi_{j}\right)_{j \in K}\right)$ feasible for GMWD, we obtain

$$
\begin{array}{cc}
f_{i}(x)-\lambda_{i} g_{i}(x) \leqq 0 & \text { for each } i \in \mathcal{N}, \\
f_{i_{0}}(x)-\lambda_{i_{0}} g_{i_{0}}(x)<0 & \text { for some } i_{0} \in \mathcal{N} .
\end{array}
$$

According to (75) and the feasibility of $x$ for VFP, we have

$$
\delta_{J_{0}}^{T} h_{J_{0}}(x) \leqq 0 .
$$

Combining (82), (83), (84), and (72), we get

$$
\begin{aligned}
& f_{i}(x)-\lambda_{i} g_{i}(x)+\delta_{J_{0}}^{T} h_{J_{0}}(x) \\
& \quad \leqq f_{i}(y)-\lambda_{i} g_{i}(y)+\delta_{J_{0}}^{T} h_{J_{0}}(y) \quad \text { for each } i \in \mathcal{N} \\
& f_{i_{0}}(x)-\lambda_{i_{0}} g_{i_{0}}(x)+\delta_{J_{0}}^{T} h_{J_{0}}(x) \\
& \quad<f_{i_{0}}(y)-\lambda_{i_{0}} g_{i_{0}}(y)+\delta_{J_{0}}^{T} h_{J_{0}}(y) \text { for some } i_{0} \in \mathcal{N} .
\end{aligned}
$$

Since $\mu_{i}>0$ for any $i \in \mathcal{N}$, by (85), (86) and (74), we obtain

$$
\begin{aligned}
\sum_{i=1}^{N} \mu_{i}\left[f_{i}(x)-\lambda_{i} g_{i}(x)\right]+\sum_{j \in J_{0}} \delta_{j} h_{j}(x) \\
<\sum_{i=1}^{N} \mu_{i}\left[f_{i}(y)-\lambda_{i} g_{i}(y)\right]+\sum_{j \in J_{0}} \delta_{j} h_{j}(y),
\end{aligned}
$$

where by using the reverse implication in (79) it follows that

$$
\begin{aligned}
& \sum_{i=1}^{N} \mu_{i} {\left[\left(d f_{i}\right)^{+}\left(y, \eta_{i}(x, y)\right)-\lambda_{i}\left(d g_{i}\right)^{+}\left(y, \eta_{i}(x, y)\right)\right] } \\
&+\sum_{j \in J_{0}} \delta_{j}\left(d h_{j}\right)^{+}\left(y, \phi_{j}(x, y)\right)<0 .
\end{aligned}
$$

Now, from (88) and (71) we obtain

$$
\sum_{s=1}^{\alpha} \sum_{j \in J_{s}} \delta_{j}\left(d h_{j}\right)^{+}\left(y, \phi_{j}(x, y)\right)>0,
$$

which is a contradiction to (81).

The proofs of parts (b) and (c) are very similar to the proof of part (a), except that, for part (b), since $\mu \geq 0$, then inequality $(87)$ becomes nonstrict $(\leqq)$ and it follows that inequalities (88) and (89) remain true and strict $(<)$ and $(>)$, respectively. 
For part (c), inequality (81) becomes strict $(<)$. Since $\mu \geq$ 0 , then inequality (87) becomes nonstrict $(\leqq)$ and it follows that the inequalities (88) and (89) become nonstrict $(\leqq)$ and $(\geqq)$, respectively. In the two cases, inequalities (89) and (81) contradict each other always. This completes the proof of the theorem.

Now we establish the following strong duality result between VFP and GMWD.

Theorem 31 (strong duality). Let $x_{0}$ be a weakly efficient solution for VFP and suppose that conditions (ii) and (iii) of Theorem 20 are satisfied. Assume also that the function $h$ satisfies the semiconstraint qualification at $x_{0}$ with respect to $\left(\phi_{j}\right)_{j \in J\left(x_{0}\right)}$. Then there exist $\mu \in \mathbb{R}_{\geq}^{N}, \lambda^{0} \in \mathbb{R}_{\geqq}^{N}$, and $\delta \in \mathbb{R}_{\geqq}^{k}$ such that $\left(x_{0}, \mu, \lambda^{0}, \delta,\left(\eta_{i}\right)_{i \in \mathcal{N}},\left(\phi_{j}\right)_{j \in K}\right) \in Y$ and the objective functions of VFP and GMWD have the same values at $x_{0}$ and $\left(x_{0}, \mu, \lambda^{0}, \delta,\left(\eta_{i}\right)_{i \in \mathcal{N}},\left(\phi_{j}\right)_{j \in K}\right)$, respectively. If, further, the weak duality between VFP and GMWD in Theorem 30 holds, then $\left(x_{0}, \mu, \lambda^{0}, \delta,\left(\eta_{i}\right)_{i \in \mathcal{N}},\left(\phi_{j}\right)_{j \in K}\right) \in Y$ is an efficient solution of GMWD.

Proof. By Theorem 20, there exist $\mu \in \mathbb{R}_{\geq}^{N}, \lambda^{0} \in \mathbb{R}_{\geqq}^{N}$, and $\delta \in \mathbb{R}_{\geqq}^{I}$ such that

$$
\begin{gathered}
\sum_{i=1}^{N} \mu_{i}\left[\left(d f_{i}\right)^{+}\left(x_{0}, \eta_{i}\left(x, x_{0}\right)\right)-\lambda_{i}^{0}\left(d g_{i}\right)^{+}\left(x_{0}, \eta_{i}\left(x, x_{0}\right)\right)\right] \\
+\sum_{j \in J\left(x_{0}\right)} \delta_{j}\left(d h_{j}\right)^{+}\left(x_{0}, \phi_{j}\left(x, x_{0}\right)\right) \geqq 0, \quad \forall x \in X,
\end{gathered}
$$

with $\lambda_{i}^{0}=f_{i}\left(x_{0}\right) / g_{i}\left(x_{0}\right), i \in \mathcal{N}$. The vector $\mu$ may be normalized according to $\mu e=1, \mu \geq 0$. By setting for all $j \in K-J\left(x_{0}\right), \delta_{j}=0$ and $\phi_{j} \equiv 0$, we obtain $\delta_{j} h_{j}\left(x_{0}\right)=0$ for all $j \in K$ and it follows that $\left(x_{0}, \mu, \lambda^{0}, \delta,\left(\eta_{i}\right)_{i \in \mathscr{N}},\left(\phi_{j}\right)_{j \in K}\right) \in$ $Y$. Clearly the objective functions of VFP and GMWD have the same values at $x_{0}$ and $\left(x_{0}, \mu, \lambda^{0}, \delta,\left(\eta_{i}\right)_{i \in \mathcal{N}},\left(\phi_{j}\right)_{j \in K}\right)$, respectively.

Next, suppose that $\left(x_{0}, \mu, \lambda^{0}, \delta,\left(\eta_{i}\right)_{i \in \mathcal{N}},\left(\phi_{j}\right)_{j \in K}\right) \in Y$ is not an efficient solution of GMWD. Then there exists $\left(\bar{y}, \bar{\mu}, \bar{\lambda}, \bar{\delta},\left(\bar{\eta}_{i}\right)_{i \in \mathcal{N}},\left(\bar{\phi}_{j}\right)_{j \in K}\right) \in Y$ such that $\lambda^{0}=$ $\left(f_{1}\left(x_{0}\right) / g_{1}\left(x_{0}\right), \ldots, f_{N}\left(x_{0}\right) / g_{N}\left(x_{0}\right)\right) \leq \bar{\lambda}=\left(\bar{\lambda}_{1}, \ldots, \bar{\lambda}_{N}\right)$, which contradicts the weak duality Theorem 30 . Hence $\left(x_{0}, \mu, \lambda^{0}, \delta,\left(\eta_{i}\right)_{i \in \mathcal{N}},\left(\phi_{j}\right)_{j \in K}\right) \in Y$ is indeed an efficient solution of GMWD.

\section{Conclusion}

In this paper, we have defined new concepts of semilocally V-type I-preinvex functions to study efficiency and duality for constrained fractional multiobjective programming. New Fritz John type necessary and Karush-Kuhn-Tucker type necessary and sufficient efficiency conditions are obtained for a feasible point to be weakly efficient or efficient under various types of generalized semilocally V-type I-preinvex requirements. Furthermore, a general Mond-Weir dual is formulated and weak and strong duality results are proved.
The results obtained in this paper generalize and extend previously known results in this area.

\section{Conflict of Interests}

The authors declare that there is no conflict of interests regarding the publication of this paper.

\section{Acknowledgment}

The authors are grateful to the anonymous referee for his/her careful reading of the paper and for his/her valuable suggestions and comments which have helped to improve the paper.

\section{References}

[1] X. Chen, "Optimality and duality for the multiobjective fractional programming with the generalized $(F, \rho)$ convexity," Journal of Mathematical Analysis and Applications, vol. 273, no. 1, pp. 190-205, 2002.

[2] G. S. Kim, M. Hee Kim, and G. M. Lee, "On optimality and duality for nonsmooth multiobjective fractional optimization problems," Nonlinear Analysis: Theory, Methods and Applications, vol. 63, no. 5-7, pp. e1867-e1876, 2005.

[3] H. Kuk, G. M. Lee, and T. Tanino, "Optimality and duality for nonsmooth multiobjective fractional programming with generalized invexity," Journal of Mathematical Analysis and Applications, vol. 262, no. 1, pp. 365-375, 2001.

[4] S. K. Mishra and J. S. Rautela, "On nonlinear multiple objective fractional programming involving semilocally type-I univex functions," Optimization Letters, vol. 3, no. 2, pp. 171-185, 2009.

[5] S. K. Mishra, S. Y. Wang, and K. K. Lai, "Multiple objective fractional programming involving semilocally type I-preinvex and related functions," Journal of Mathematical Analysis and Applications, vol. 310, no. 2, pp. 626-640, 2005.

[6] C. Niculescu, "Optimality and duality in multiobjective fractional programming involving $\rho$-semilocally type I-preinvex and related functions," Journal of Mathematical Analysis and Applications, vol. 335, no. 1, pp. 7-19, 2007.

[7] V. Preda, "Optimality and duality in fractional multiple objective programming involving semilocally preinvex and related functions," Journal of Mathematical Analysis and Applications, vol. 288, no. 2, pp. 365-382, 2003.

[8] M. Soleimani-damaneh, "Optimality for nonsmooth fractional multiple objective programming," Nonlinear Analysis: Theory, Methods \& Applications A: Theory and Methods, vol. 68, no. 10, pp. 2873-2878, 2008.

[9] V. Preda, I. M. Stancu-Minasian, M. Beldiman, and A.-M. Stancu, "On a general duality model in multiobjective fractional programming with $n$-set functions," Mathematical and Computer Modelling, vol. 54, no. 1-2, pp. 490-496, 2011.

[10] R. U. Verma, "Role of $(\rho, \eta, A)$-invexity to $\epsilon$-optimality conditions for multiple objective fractional programming," Applied Mathematics and Computation, vol. 218, no. 17, pp. 8444-8452, 2012.

[11] G. J. Zalmai, "Efficiency conditions and duality models for multiobjective fractional subset programming problems with generalized $(F, \alpha, \rho, \theta)-V$-convex functions," Computers \& Mathematics with Applications, vol. 43, no. 12, pp. 1489-1520, 2002.

[12] G. J. Zalmai, "Generalized $(F, b, \phi, \rho, \theta)$-univex $n$-set functions and global semiparametric sufficient efficiency conditions in 
multiobjective fractional subset programming," International Journal of Mathematics and Mathematical Sciences, vol. 2005, no. 6, pp. 949-973, 2005.

[13] G. J. Zalmai, "Generalized $(F, b, \phi, \rho, \theta)$-univex $n$-set functions and semiparametric duality models in multiobjective fractional subset programming," International Journal of Mathematics and Mathematical Sciences, vol. 2005, no. 7, pp. 1109-1133, 2005.

[14] T. R. Gulati and Geeta, "Duality in nondifferentiable multiobjective fractional programming problem with generalized invexity," Journal of Applied Mathematics and Computing, vol. 35, no. 1-2, pp. 103-118, 2011.

[15] S. K. Suneja, M. K. Srivastava, and M. Bhatia, "Higher order duality in multiobjective fractional programming with support functions," Journal of Mathematical Analysis and Applications, vol. 347, no. 1, pp. 8-17, 2008.

[16] T. Antczak, "A modified objective function method for solving nonlinear multiobjective fractional programming problems," Journal of Mathematical Analysis and Applications, vol. 322, no. 2, pp. 971-989, 2006.

[17] A. Chinchuluun, D. Yuan, and P. M. Pardalos, "Optimality conditions and duality for nondifferentiable multiobjective fractional programming with generalized convexity," Annals of Operations Research, vol. 154, pp. 133-147, 2007.

[18] J.-C. Liu and C.-Y. Liu, "Optimality and duality for multiobjective fractional programming involving nonsmooth generalized $(F, b, \phi, \rho, \theta)$-univex functions," International Journal of Mathematics and Mathematical Sciences, vol. 2013, Article ID 284046, 10 pages, 2013.

[19] S. K. Mishra, S. Wang, and K. K. Lai, V-Invex Functions and Vector Optimization, Springer Science + Busineess Media, New York, NY, USA, 2008.

[20] R. U. Verma, "Weak $\epsilon$-efficiency conditions for multiobjective fractional programming," Applied Mathematics and Computation, vol. 219, no. 12, pp. 6819-6827, 2013.

[21] X. Zhang and Z. Wu, "Optimality conditions and duality of three kinds of nonlinear fractional programming problems," Advances in Operations Research, vol. 2013, Article ID 708979, 9 pages, 2013.

[22] M. A. Hanson, "On sufficiency of the Kuhn-Tucker conditions," Journal of Mathematical Analysis and Applications, vol. 80, no. 2, pp. 545-550, 1981.

[23] F. Dinuzzo, C. S. Ong, P. Gehler, and G. Pillonetto, "Learning output kernels with block coordinate descent," in Proceedings of the 28th International Conference on Machine Learning, pp. 4956, Bellevue, Wash, USA, July 2011.

[24] H. Nickisch and M. Seeger, "Multiple kernel learning: a unifying probabilistic viewpoint," version 3, http://arxiv.org/abs/ 1103.0897v3, 2012.

[25] G. M. Ewing, "Sufficient conditions for global minima of suitably convex functionals from variational and control theory," SIAM Review, vol. 19, no. 2, pp. 202-220, 1977.

[26] R. N. Kaul and S. Kaur, "Generalizations of convex and related functions," European Journal of Operational Research, vol. 9, no. 4, pp. 369-377, 1982.

[27] R. N. Kaul and S. Kaur, "Sufficient optimality conditions using generalized convex functions," Opsearch, vol. 19, no. 4, pp. 212224, 1982.

[28] V. Preda, "Optimality conditions and duality in multiple objective programming involving semilocally convex and related functions," Optimization, vol. 36, no. 3, pp. 219-230, 1996.
[29] V. Preda and I. M. Stancu-Minasian, "Duality in multiple objective programming involving semilocally preinvex and related functions," Glasnik Matematički. Serija III, vol. 32, pp. 153-165, 1997.

[30] V. Preda, I. M. Stancu-Minasian, and A. Batatorescu, “Optimality and duality in nonlinear programming involving semilocally preinvex and related functions," Journal of Information \& Optimization Sciences, vol. 17, no. 3, pp. 585-596, 1996.

[31] I. M. Stancu-Minasian, "Optimality conditions and duality in fractional programming involving semilocally preinvex and related functions," Journal of Information \& Optimization Sciences, vol. 23, no. 1, pp. 185-201, 2002.

[32] H. Slimani and M. S. Radjef, "Duality for nonlinear programming under generalized Kuhn-Tucker condition," International Journal of Optimization: Theory Methods and Applications, vol. 1, no. 1, pp. 75-86, 2009.

[33] H. Slimani and M. S. Radjef, Multiobjective Programming under Generalized Invexity: Optimality, Duality, Applications, LAP Lambert Academic Publishing, Saarbrücken, Germany, 2010.

[34] H. Slimani and M. S. Radjef, "Nondifferentiable multiobjective programming under generalized $d_{I}$-invexity," European Journal of Operational Research, vol. 202, no. 1, pp. 32-41, 2010.

[35] I. Ahmad, "Efficiency and duality in nondifferentiable multiobjective programming involving directional derivative," Applied Mathematics, vol. 2, no. 4, pp. 452-460, 2011.

[36] M. Arana-Jiménez, G. Ruiz-Garzón, R. Osuna-Gómez, and B. Hernández-Jiménez, "Duality and a characterization of pseudoinvexity for Pareto and weak Pareto solutions in nondifferentiable multiobjective programming," Journal of Optimization Theory and Applications, vol. 156, no. 2, pp. 266-277, 2013.

[37] K. H. Elster and R. Nehse, Optimality Conditions for Some Nonconvex Problems, Springer, New York, NY, USA, 1980.

[38] Y. L. Ye, “ $d$-invexity and optimality conditions," Journal of Mathematical Analysis and Applications, vol. 162, no. 1, pp. 242249, 1991.

[39] M. Hayashi and H. Komiya, "Perfect duality for convexlike programs," Journal of Optimization Theory and Applications, vol. 38, no. 2, pp. 179-189, 1982. 


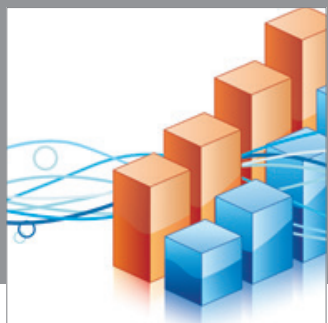

Advances in

Operations Research

mansans

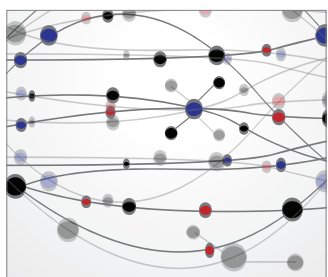

The Scientific World Journal
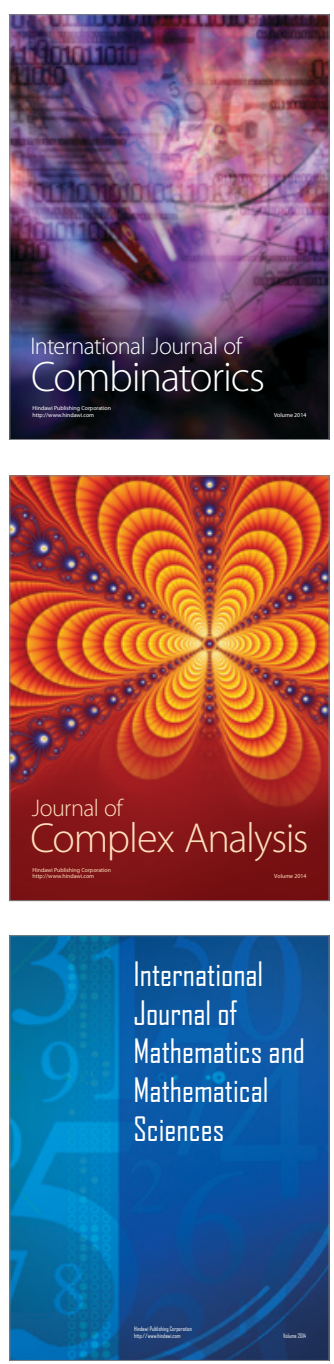
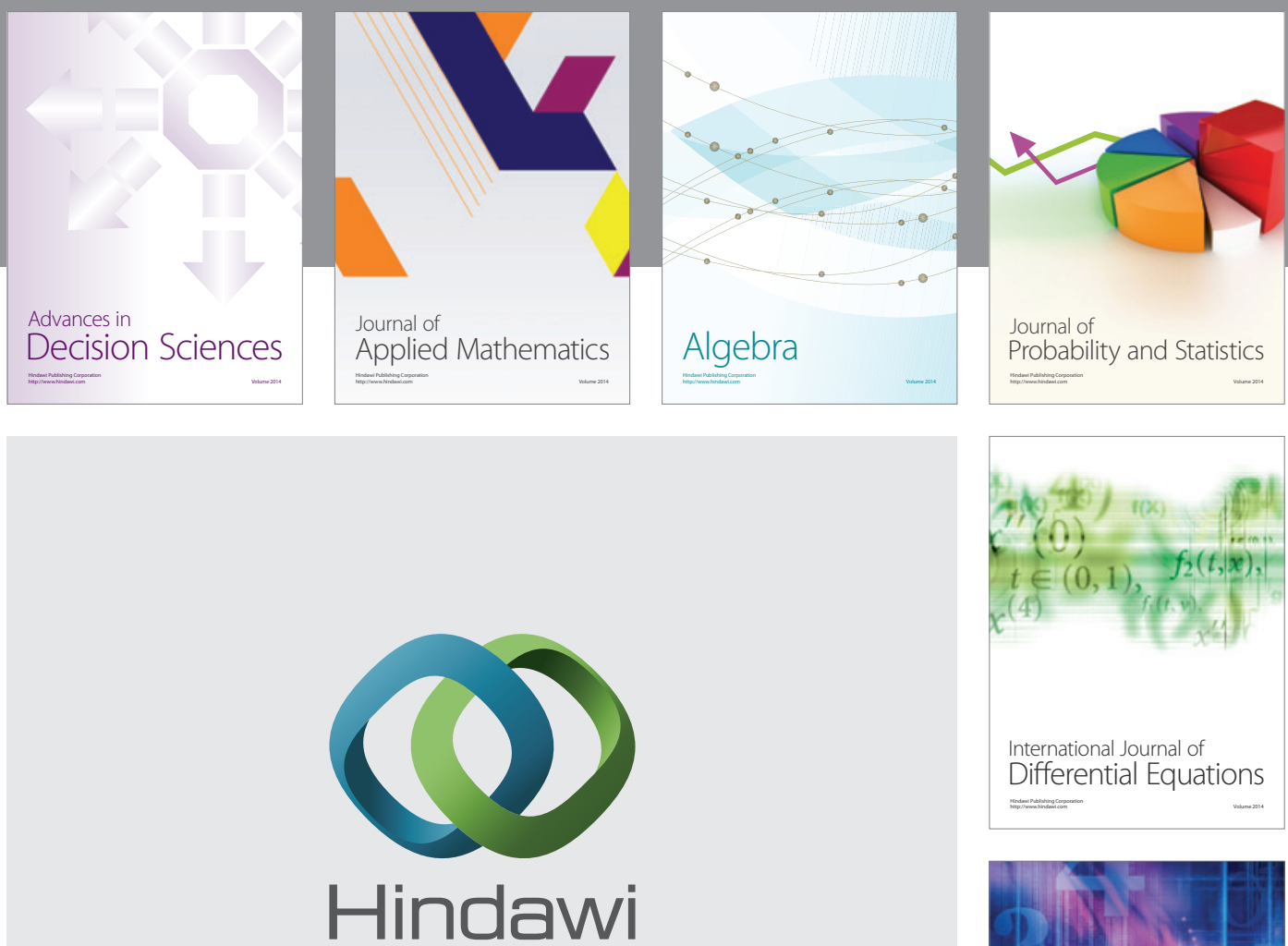

Submit your manuscripts at http://www.hindawi.com
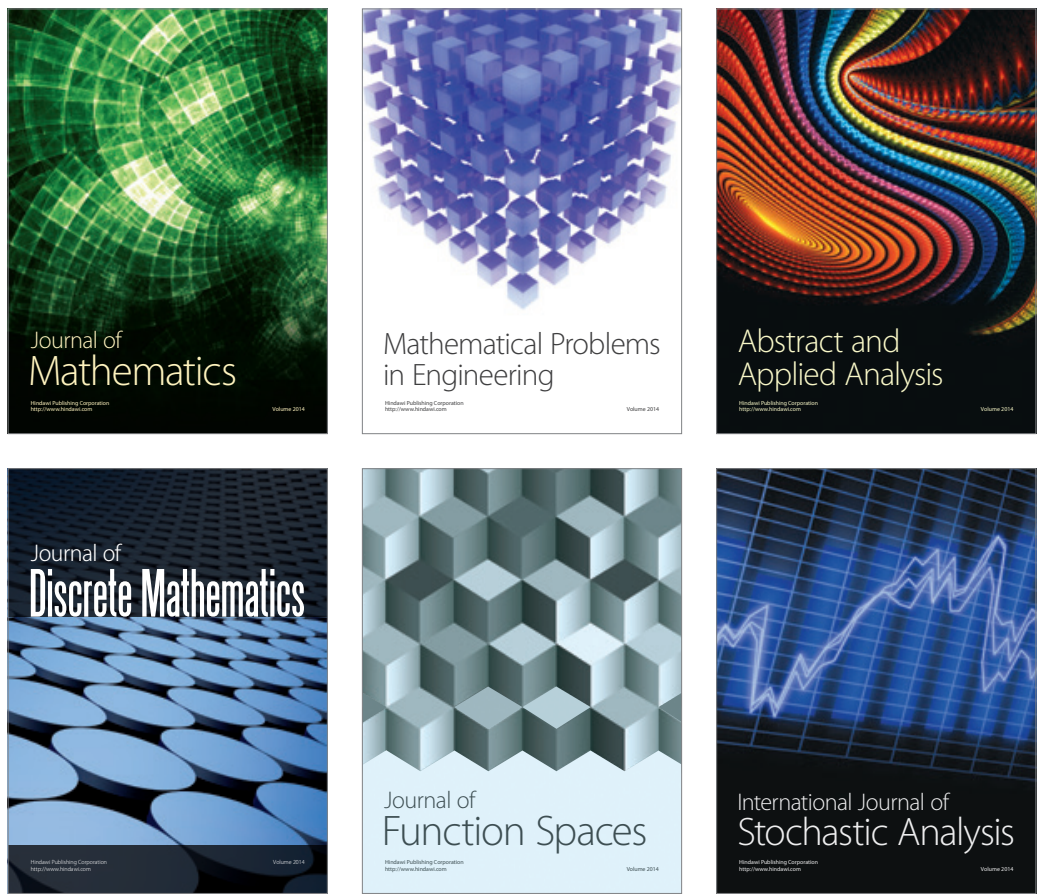

Journal of

Function Spaces

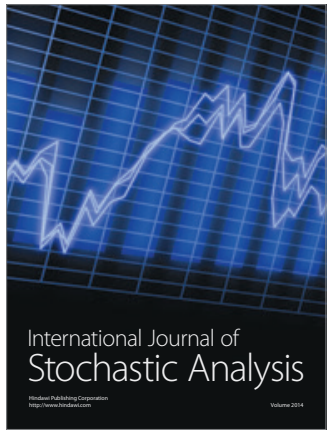

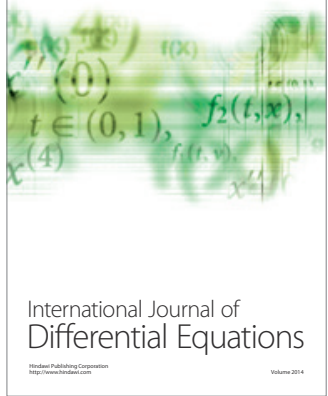
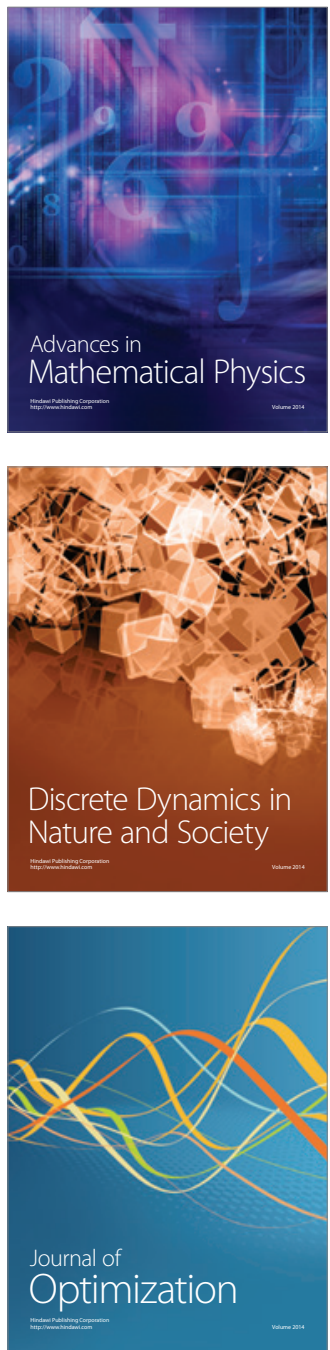